\title{
Vibrationally Resolved Absorption and Luminescence Spectra of Mass-selected Free-base and Zinc Phthalocyanine Radical Cations Isolated in Solid Ne
}

Karolina A. Haupa, ${ }^{1 *}$ Niklas P. Krappel, ${ }^{1}$ Dmitry Strelnikov, ${ }^{1}$ and Manfred M. Kappes ${ }^{1,2^{*}}$

${ }^{1}$ Institute of Physical Chemistry II, KIT, Fritz-Haber-Weg 2, 76131 Karlsruhe, Germany.

${ }^{2}$ Institute of Nanotechnology, KIT, Hermann-von-Helmholtz-Platz 1, 76344 Eggenstein-Leopoldshafen, Germany.

Corresponding authors:

*E-mail: karolina.haupa@gmail.com

*E-mail: manfred.kappes@kit.edu 


\section{Table of contents}

1. Table S1. Vibrational frequencies in $\mathrm{cm}^{-1}$ of the ground states and the first excited states of $\mathrm{H}_{2} \mathrm{Pc}$, $\mathrm{H}_{2} \mathrm{Pc}^{+}, \mathrm{ZnPc}$, and $\mathrm{ZnPc} \mathrm{C}^{+}$calculated with the (TD)-B3LYP/cc-pVDZ method.

2. Figure S1. Optimized geometries of ground states of $\mathrm{H}_{2} \mathrm{Pc}(\mathrm{a}), \mathrm{H}_{2} \mathrm{Pc}^{+}(\mathrm{b}), \mathrm{ZnPc}(\mathrm{c})$, and $\mathrm{ZnPc}^{+}(\mathrm{d})$ calculated with the B3LYP/cc-pVDZ method.

3. Figure S2. Absorption spectra recorded for $\mathrm{ZnPc}^{+} / \mathrm{Ne}, \mathrm{ZnPc}^{+} / \mathrm{CH}_{3} \mathrm{Cl} / \mathrm{Ne}$, and $\mathrm{ZnPc}^{+} / \mathrm{SF}_{6} / \mathrm{Ne}$ at $5 \mathrm{~K}$.

4. Figure S3. Comparison of the absorption spectrum of $\mathrm{ZnPc}$ recorded in this work with previous studies in $\mathrm{Ne}, \mathrm{Ar}$, and $\mathrm{N}_{2}$ matrices.

5. Table S2. Band maxima $\left( \pm 1 \mathrm{~nm}\right.$ ) of the $\mathrm{S}_{0} \rightarrow \mathrm{S}_{1}$ absorption of $\mathrm{ZnPc}$, isolated in solid $\mathrm{Ne}$ at $5 \mathrm{~K}$, compared with literature data acquired in other matrix isolation experiments and in gas-phase.

6. Table S3. Observed band maxima in the absorption spectra of $\mathrm{H}_{2} \mathrm{Pc}$ isolated in solid $\mathrm{Ne}$ at $5 \mathrm{~K}$.

7. Figure S4. Comparison of absorption and reversed emission (532 nm excitation) spectra of $\mathrm{H}_{2} \mathrm{Pc}$ isolated in solid Ne.

8. Table S4. Vertical excitation wavelengths and oscillator strengths of $\mathrm{ZnPc}, \mathrm{ZnPc}^{+}, \mathrm{H}_{2} \mathrm{Pc}$, and $\mathrm{H}_{2} \mathrm{Pc}^{+}$ electronic transitions calculated with the TD-B3LYP/cc-pVDZ method.

9. Figure S5. Comparison of the $\mathrm{ZnPc}$ absorption spectrum (recorded in a $\mathrm{ZnPc}{ }^{+} / \mathrm{CH}_{3} \mathrm{Cl} / \mathrm{Ne}$ matrix) with predicted spectral pattern on the basis of our FC and FCHT TD-B3LYP/cc-pVDZ calculations

10. Figure S6. Comparison of the vibrational pattern associated with the $\mathrm{D}_{0} \rightarrow \mathrm{D}_{1}$ transition of $\mathrm{ZnPc}^{+}$ isolated in solid $\mathrm{Ne}\left(0-0\right.$ origin band at $\left.12153 \mathrm{~cm}^{-1}\right)$ with the predicted Franck-Condon (FC) and Franck-Condon Hertzberg-Teller (FCHT) spectra based on TD-B3LYP/cc-pVDZ.

11. Table S5. $\mathrm{S}_{1} \rightarrow \mathrm{S}_{0}$ fluorescence of $\mathrm{ZnPc}$ isolated in solid $\mathrm{Ne}$ at $5 \mathrm{~K}$ as measured in this work, compared with literature data acquired in other matrix isolation experiments and in STM-LE measurements.

12. Table S6. $\mathrm{S}_{1} \rightarrow \mathrm{S}_{0}$ fluorescence of $\mathrm{H}_{2} \mathrm{Pc}$ isolated in solid $\mathrm{Ne}$ at $5 \mathrm{~K}$, compared with literature data acquired in other matrix isolation experiments and in gas-phase; wavenumbers in respect to $0-0$ band origin.

13. Figure S7. Comparison of the $\mathrm{ZnPc}$ LIF spectrum recorded in this work (in a $\mathrm{ZnPc}+\mathrm{CH}_{3} \mathrm{Cl} / \mathrm{Ne}$ matrix) with the predicted spectral pattern on the basis of our FC and FCHT TD-B3LYP/cc-pVDZ calculations.

14. Figure S8. Comparison of the $\mathrm{H}_{2} \mathrm{PC}$ LIF spectrum recorded in this work (in a $\mathrm{H}_{2} \mathrm{Pc}^{+} / \mathrm{CH}_{3} \mathrm{Cl} / \mathrm{Ne}$ matrix) with predicted spectral pattern on the basis of our FC and FCHT TD-B3LYP/cc-pVDZ calculations.

15. Table S7. $\mathrm{S}_{1} \rightarrow \mathrm{S}_{0}$ fluorescence of $\mathrm{ZnPc}^{+}$. Comparison of vibrational features observed in the LIF spectra of matrix isolated $\mathrm{ZnPc}^{+}$with those acquired in STM-LE measurements and predictions of TDB3LYP/cc-pVDZ calculations at the FC and FCHT levels.

16. Table S8. $\mathrm{S}_{1} \rightarrow \mathrm{S}_{0}$ fluorescence of $\mathrm{H}_{2} \mathrm{Pc}$. Comparison with (i) STM-LE measurements and (ii) predictions of TD-B3LYP/cc-pVDZ calculations at FC and FCHT levels.

17. Figure S9. Comparison of the LIF spectrum of $\mathrm{H}_{2} \mathrm{Pc}^{+}$recorded in a $\mathrm{H}_{2} \mathrm{Pc}^{+} / \mathrm{CH}_{3} \mathrm{Cl} / \mathrm{Ne}$ matrix $(785 \mathrm{~nm}$ excitation) with TD-B3LYP/cc-pVDZ (FCHT) and TD-B3PW91/cc-pVDZ (FC) predictions.

18. Table S9. $\mathrm{D}_{1} \rightarrow \mathrm{D}_{0}$ fluorescence of $\mathrm{H}_{2} \mathrm{Pc}^{+}$. Tabulation of vibrotational features observed in the LIF spectrum of matrix isolated $\mathrm{H}_{2} \mathrm{Pc}^{+}$.

19. Table S10. $D_{1} \rightarrow D_{0}$ fluorescence of $\mathrm{H}_{2} \mathrm{PC}^{+}$. Tabulation of vibrational features predicted by FC and FCHT TD-B3LYP/cc-pVDZ method simulations.

20. Figure S10. Comparison of the LIF spectrum of $\mathrm{H}_{2} \mathrm{Pc}^{+}$recorded in different experiments.

21. Table S11. Comparison of the band origins of $\mathrm{ZnPc}, \mathrm{ZnPc}{ }^{+}, \mathrm{H}_{2} \mathrm{Pc}$, and $\mathrm{H}_{2} \mathrm{Pc}^{+}$as observed here in absorption and emission spectra with predictions from TD-B3LYP/cc-pVDZ method calculations. 
Table S1. Vibrational frequencies in $\mathrm{cm}^{-1}$ of the ground states and the first excited states of $\mathrm{H}_{2} \mathrm{Pc}_{2} \mathrm{H}_{2} \mathrm{Pc}^{+}$, $\mathrm{ZnPc}$, and $\mathrm{ZnPc}{ }^{+}$calculated with the (TD)-B3LYP/cc-pVDZ method (harmonic approximation).

\begin{tabular}{|c|c|c|c|c|c|c|c|c|}
\hline \multirow[b]{2}{*}{ mode } & \multicolumn{2}{|c|}{$\mathrm{H}_{2} \mathrm{Pc}$} & \multicolumn{2}{|c|}{$\mathrm{H}_{2} \mathrm{Pc}^{+}$} & \multicolumn{2}{|c|}{$\mathrm{ZnPc}$} & \multicolumn{2}{|c|}{$\mathrm{ZnPc}^{+}$} \\
\hline & So & $S_{1}$ & $D_{0}$ & $\mathrm{D}_{1}$ & So & $S_{1}$ & $\mathrm{D}_{0}$ & $\mathrm{D}_{1}$ \\
\hline$v 1$ & 19.2 & 16.3 & 15.6 & 17.8 & 21.9 & 16.7 & 18.0 & 7.1 \\
\hline$v 2$ & 37.4 & 28.8 & 18.2 & 37.7 & 30.5 & 29.4 & 29.1 & 28.5 \\
\hline$v 3$ & 40.5 & 36.8 & 37.1 & 38.0 & 60.0 & 52.4 & 54.5 & 45.2 \\
\hline$v 4$ & 53.2 & 51.0 & 51.4 & 52.6 & 60.0 & 53.0 & 54.5 & 47.6 \\
\hline$v 5$ & 57.9 & 52.0 & 52.1 & 56.1 & 63.5 & 61.7 & 62.6 & 61.1 \\
\hline$v 6$ & 80.0 & 76.8 & 81.7 & 84.2 & 112.6 & 112.2 & 112.8 & 109.0 \\
\hline$v 7$ & 116.0 & 114.6 & 111.0 & 109.8 & 119.8 & 119.1 & 117.9 & 110.7 \\
\hline v8 & 120.4 & 120.1 & 118.3 & 118.9 & 119.8 & 119.4 & 119.5 & 116.2 \\
\hline v9 & 122.0 & 121.1 & 120.2 & 120.8 & 123.0 & 119.6 & 119.5 & 118.9 \\
\hline$v 10$ & 123.5 & 121.6 & 121.8 & 122.8 & 124.8 & 120.3 & 120.0 & 119.0 \\
\hline$v 11$ & 127.9 & 128.1 & 124.7 & 125.8 & 126.4 & 122.6 & 123.4 & 120.7 \\
\hline$v 12$ & 132.3 & 131.1 & 132.0 & 130.7 & 126.4 & 124.2 & 123.4 & 120.8 \\
\hline$v 13$ & 137.0 & 131.6 & 132.7 & 135.4 & 134.0 & 126.9 & 128.1 & 125.0 \\
\hline$v 14$ & 177.9 & 165.9 & 176.6 & 180.1 & 156.4 & 155.7 & 158.2 & 157.6 \\
\hline$v 15$ & 208.1 & 206.4 & 195.0 & 207.9 & 210.0 & 208.4 & 208.9 & 193.2 \\
\hline$v 16$ & 213.4 & 207.5 & 207.0 & 210.3 & 228.9 & 214.6 & 222.2 & 205.9 \\
\hline$v 17$ & 223.3 & 215.7 & 215.2 & 214.8 & 231.5 & 219.8 & 225.3 & 206.1 \\
\hline$v 18$ & 225.0 & 220.1 & 217.9 & 216.3 & 240.7 & 222.1 & 227.0 & 214.3 \\
\hline$v 19$ & 229.8 & 227.1 & 226.4 & 229.5 & 240.7 & 225.7 & 227.0 & 215.8 \\
\hline$v 20$ & 238.9 & 229.3 & 229.6 & 231.2 & 248.4 & 230.6 & 229.5 & 225.3 \\
\hline$v 21$ & 263.1 & 239.9 & 235.6 & 253.1 & 248.4 & 247.0 & 248.0 & 242.6 \\
\hline$v 22$ & 272.1 & 265.5 & 260.4 & 262.0 & 254.3 & 247.8 & 249.8 & 246.9 \\
\hline$v 23$ & 273.7 & 266.0 & 272.1 & 273.1 & 254.4 & 248.4 & 249.8 & 249.4 \\
\hline$v 24$ & 281.6 & 272.1 & 278.4 & 280.2 & 258.7 & 258.4 & 258.5 & 257.6 \\
\hline$v 25$ & 289.1 & 277.5 & 279.5 & 281.6 & 289.8 & 267.3 & 278.2 & 258.3 \\
\hline$v 26$ & 340.8 & 322.6 & 322.3 & 324.7 & 289.8 & 286.8 & 278.2 & 279.8 \\
\hline$v 27$ & 435.3 & 424.4 & 421.1 & 405.6 & 305.7 & 303.4 & 305.8 & 304.5 \\
\hline$v 28$ & 435.8 & 425.5 & 422.7 & 409.0 & 305.7 & 305.2 & 305.8 & 306.0 \\
\hline$v 29$ & 436.1 & 431.2 & 425.0 & 426.9 & 351.3 & 336.6 & 332.7 & 323.4 \\
\hline v30 & 445.0 & 436.0 & 435.8 & 432.6 & 438.4 & 425.9 & 423.8 & 415.2 \\
\hline v31 & 449.3 & 439.8 & 449.4 & 443.5 & 438.5 & 427.9 & 426.5 & 417.4 \\
\hline$v 32$ & 486.2 & 454.0 & 484.0 & 464.7 & 438.5 & 433.3 & 426.5 & 419.3 \\
\hline v33 & 496.8 & 488.1 & 493.1 & 487.5 & 450.1 & 441.5 & 438.0 & 429.3 \\
\hline v34 & 501.7 & 493.5 & 495.5 & 496.2 & 451.4 & 442.5 & 454.0 & 437.1 \\
\hline v35 & 508.2 & 496.4 & 498.5 & 504.5 & 488.8 & 451.2 & 486.7 & 446.7 \\
\hline v36 & 508.3 & 498.1 & 501.4 & 504.6 & 510.6 & 492.9 & 500.3 & 484.4 \\
\hline v37 & 535.2 & 520.0 & 519.9 & 511.8 & 510.6 & 501.9 & 500.3 & 493.7 \\
\hline v38 & 550.2 & 548.6 & 549.6 & 546.1 & 512.3 & 504.2 & 509.6 & 502.8 \\
\hline v39 & 557.3 & 553.7 & 555.4 & 555.5 & 512.3 & 510.8 & 509.6 & 507.6 \\
\hline v40 & 565.2 & 562.1 & 559.3 & 558.2 & 537.5 & 520.1 & 520.8 & 509.3 \\
\hline v41 & 576.1 & 568.7 & 562.3 & 559.2 & 560.5 & 559.1 & 559.9 & 556.6 \\
\hline$v 42$ & 576.4 & 573.6 & 570.0 & 570.8 & 582.6 & 577.4 & 578.3 & 568.5 \\
\hline v43 & 586.9 & 577.0 & 575.7 & 574.1 & 582.6 & 577.5 & 578.3 & 570.1 \\
\hline$v 44$ & 609.0 & 606.0 & 607.9 & 575.0 & 585.6 & 580.6 & 579.0 & 573.2 \\
\hline$v 45$ & 629.3 & 625.9 & 625.1 & 610.2 & 598.9 & 596.0 & 598.7 & 595.0 \\
\hline$v 46$ & 629.6 & 627.3 & 627.4 & 620.4 & 624.8 & 622.1 & 625.2 & 621.7 \\
\hline$v 47$ & 637.6 & 630.7 & 645.6 & 625.0 & 641.8 & 636.3 & 646.9 & 640.6 \\
\hline$v 48$ & 653.1 & 638.9 & 664.1 & 639.3 & 649.3 & 645.8 & 646.9 & 642.0 \\
\hline$v 49$ & 662.3 & 658.3 & 669.5 & 653.4 & 649.3 & 646.2 & 650.2 & 645.7 \\
\hline$v 50$ & 690.1 & 686.6 & 686.5 & 677.2 & 662.1 & 646.4 & 671.3 & 649.5 \\
\hline v51 & 693.6 & 687.2 & 692.3 & 681.1 & 662.1 & 660.2 & 671.3 & 672.0 \\
\hline$v 52$ & 702.0 & 690.7 & 708.2 & 683.2 & 688.5 & 684.9 & 684.6 & 682.7 \\
\hline$v 53$ & 705.7 & 695.6 & 714.7 & 696.0 & 696.4 & 689.4 & 695.0 & 683.7 \\
\hline$v 54$ & 733.7 & 721.9 & 736.0 & 723.1 & 706.2 & 693.0 & 711.0 & 697.2 \\
\hline$v 55$ & 739.5 & 732.0 & 739.8 & 732.7 & 715.8 & 705.2 & 725.6 & 716.5 \\
\hline
\end{tabular}




\begin{tabular}{|c|c|c|c|c|c|c|c|c|}
\hline v56 & 741.9 & 733.1 & 740.2 & 736.8 & 741.3 & 730.6 & 748.7 & 738.1 \\
\hline v57 & 742.6 & 734.9 & 743.5 & 740.1 & 741.3 & 732.5 & 748.7 & 740.5 \\
\hline v58 & 743.2 & 739.6 & 743.9 & 741.3 & 751.4 & 741.3 & 758.2 & 749.3 \\
\hline v59 & 748.0 & 743.3 & 749.8 & 741.5 & 762.0 & 757.1 & 760.9 & 756.2 \\
\hline$v 60$ & 780.1 & 772.3 & 770.4 & 763.3 & 764.9 & 757.3 & 761.6 & 756.8 \\
\hline$v 61$ & 781.7 & 779.6 & 781.4 & 770.5 & 764.9 & 761.0 & 761.6 & 760.2 \\
\hline$v 62$ & 791.7 & 784.2 & 799.3 & 793.9 & 789.3 & 786.8 & 787.7 & 787.3 \\
\hline$v 63$ & 793.4 & 784.9 & 800.9 & 797.6 & 793.7 & 787.8 & 803.1 & 788.4 \\
\hline$v 64$ & 797.2 & 791.6 & 802.5 & 797.6 & 796.7 & 788.1 & 806.0 & 794.6 \\
\hline$v 65$ & 800.4 & 795.9 & 803.2 & 798.6 & 796.7 & 791.2 & 806.0 & 795.2 \\
\hline$v 66$ & 804.6 & 797.0 & 805.6 & 799.1 & 800.4 & 792.8 & 811.6 & 802.2 \\
\hline$v 67$ & 805.7 & 803.5 & 811.6 & 808.2 & 815.6 & 809.9 & 815.6 & 805.1 \\
\hline$v 68$ & 812.1 & 806.0 & 812.2 & 808.4 & 815.6 & 810.4 & 815.6 & 807.1 \\
\hline$v 69$ & 814.3 & 806.4 & 812.9 & 808.7 & 818.6 & 811.3 & 816.9 & 808.9 \\
\hline$v 70$ & 815.5 & 810.4 & 815.8 & 814.0 & 818.6 & 811.6 & 820.1 & 813.8 \\
\hline$v 71$ & 818.9 & 810.5 & 819.5 & 817.6 & 819.5 & 813.8 & 820.3 & 813.9 \\
\hline$v 72$ & 819.5 & 811.0 & 819.9 & 822.0 & 819.5 & 814.3 & 820.3 & 814.0 \\
\hline$v 73$ & 834.8 & 832.9 & 831.8 & 836.7 & 852.5 & 851.8 & 853.5 & 815.0 \\
\hline$v 74$ & 850.0 & 846.8 & 849.3 & 850.9 & 861.3 & 858.7 & 857.9 & 851.8 \\
\hline$v 75$ & 890.6 & 881.7 & 889.9 & 876.9 & 903.5 & 878.4 & 902.1 & 855.3 \\
\hline$v 76$ & 899.0 & 891.9 & 894.6 & 885.1 & 903.5 & 898.9 & 902.1 & 897.9 \\
\hline$v 77$ & 906.4 & 899.9 & 919.4 & 899.6 & 907.5 & 900.9 & 920.0 & 908.7 \\
\hline$v 78$ & 906.6 & 900.0 & 919.7 & 913.5 & 907.5 & 901.4 & 920.7 & 916.7 \\
\hline$v 79$ & 906.6 & 908.8 & 920.0 & 913.6 & 907.5 & 903.7 & 920.7 & 917.2 \\
\hline$v 80$ & 906.7 & 908.9 & 920.1 & 917.5 & 907.6 & 909.9 & 921.2 & 923.6 \\
\hline$v 81$ & 976.7 & 947.3 & 989.5 & 951.4 & 957.9 & 910.1 & 956.5 & 923.7 \\
\hline v82 & 976.7 & 976.5 & 989.6 & 987.9 & 979.7 & 975.8 & 992.2 & 956.2 \\
\hline$v 83$ & 982.6 & 976.6 & 994.8 & 991.0 & 980.0 & 975.8 & 992.5 & 989.8 \\
\hline$v 84$ & 982.6 & 978.2 & 994.8 & 991.0 & 980.0 & 978.7 & 992.5 & 989.8 \\
\hline$v 85$ & 1008.4 & 978.4 & 1024.2 & 992.1 & 980.2 & 978.7 & 992.7 & 992.1 \\
\hline$v 86$ & 1008.4 & 1005.3 & 1024.2 & 1017.0 & 1010.7 & 1003.4 & 1026.5 & 992.1 \\
\hline$v 87$ & 1012.9 & 1005.3 & 1028.2 & 1022.4 & 1010.8 & 1003.4 & 1026.6 & 1020.4 \\
\hline$v 88$ & 1012.9 & 1006.5 & 1028.2 & 1024.9 & 1010.8 & 1008.1 & 1026.6 & 1020.4 \\
\hline$v 89$ & 1027.9 & 1006.5 & 1028.8 & 1024.9 & 1010.9 & 1008.1 & 1026.6 & 1025.2 \\
\hline$v 90$ & 1028.0 & 1024.6 & 1028.8 & 1029.0 & 1028.7 & 1019.2 & 1029.5 & 1025.2 \\
\hline$v 91$ & 1028.5 & 1024.6 & 1030.7 & 1030.4 & 1029.0 & 1025.9 & 1030.4 & 1026.7 \\
\hline$v 92$ & 1029.5 & 1027.2 & 1031.4 & 1031.1 & 1029.0 & 1025.9 & 1030.4 & 1027.7 \\
\hline$v 93$ & 1041.2 & 1027.6 & 1034.6 & 1041.5 & 1030.1 & 1028.9 & 1031.6 & 1028.6 \\
\hline v94 & 1060.6 & 1054.3 & 1043.0 & 1059.9 & 1053.7 & 1029.3 & 1049.2 & 1030.1 \\
\hline$v 95$ & 1061.2 & 1057.8 & 1051.5 & 1071.2 & 1081.7 & 1048.2 & 1080.5 & 1049.4 \\
\hline$v 96$ & 1087.5 & 1065.6 & 1071.3 & 1079.7 & 1081.7 & 1069.9 & 1080.5 & 1055.3 \\
\hline v97 & 1097.7 & 1075.4 & 1075.9 & 1081.0 & 1107.9 & 1080.2 & 1094.5 & 1071.2 \\
\hline$v 98$ & 1112.0 & 1094.2 & 1095.5 & 1105.2 & 1128.1 & 1088.1 & 1094.5 & 1078.8 \\
\hline$v 99$ & 1118.3 & 1104.0 & 1097.1 & 1117.7 & 1128.3 & 1101.7 & 1095.4 & 1079.0 \\
\hline$v 100$ & 1125.9 & 1105.5 & 1113.2 & 1122.0 & 1128.3 & 1114.7 & 1112.9 & 1103.3 \\
\hline$v 101$ & 1129.8 & 1125.5 & 1129.1 & 1126.3 & 1134.8 & 1137.9 & 1119.5 & 1103.6 \\
\hline$v 102$ & 1134.7 & 1129.1 & 1134.8 & 1136.6 & 1134.8 & 1138.4 & 1141.0 & 1132.8 \\
\hline$v 103$ & 1137.6 & 1129.5 & 1136.0 & 1148.5 & 1145.1 & 1140.7 & 1141.0 & 1142.5 \\
\hline$v 104$ & 1154.0 & 1146.5 & 1165.3 & 1160.4 & 1154.3 & 1142.3 & 1144.0 & 1163.7 \\
\hline$v 105$ & 1170.8 & 1155.2 & 1175.3 & 1176.5 & 1168.7 & 1149.4 & 1165.8 & 1166.4 \\
\hline$v 106$ & 1174.1 & 1166.9 & 1175.7 & 1182.1 & 1179.6 & 1155.6 & 1178.2 & 1169.5 \\
\hline$v 107$ & 1176.5 & 1169.2 & 1179.3 & 1184.2 & 1184.4 & 1172.0 & 1178.2 & 1178.5 \\
\hline$v 108$ & 1207.2 & 1172.0 & 1180.2 & 1199.4 & 1184.4 & 1174.2 & 1180.3 & 1180.1 \\
\hline$v 109$ & 1210.2 & 1199.4 & 1199.9 & 1201.7 & 1206.3 & 1175.2 & 1188.4 & 1182.5 \\
\hline$v 110$ & 1210.4 & 1202.8 & 1203.5 & 1206.1 & 1210.8 & 1195.9 & 1197.6 & 1187.8 \\
\hline$v 111$ & 1214.0 & 1205.6 & 1204.7 & 1213.9 & 1212.3 & 1198.1 & 1203.7 & 1187.8 \\
\hline$v 112$ & 1224.9 & 1212.5 & 1216.1 & 1228.5 & 1212.3 & 1205.0 & 1203.7 & 1204.1 \\
\hline$v 113$ & 1255.9 & 1238.3 & 1240.4 & 1259.4 & 1236.1 & 1206.9 & 1222.7 & 1222.4 \\
\hline$v 114$ & 1279.2 & 1265.1 & 1276.4 & 1265.6 & 1304.7 & 1289.6 & 1302.2 & 1292.9 \\
\hline$v 115$ & 1295.0 & 1290.0 & 1296.3 & 1290.7 & 1304.7 & 1298.5 & 1302.2 & 1298.0 \\
\hline
\end{tabular}




\begin{tabular}{|c|c|c|c|c|c|c|c|c|}
\hline$v 116$ & 1308.5 & 1293.4 & 1299.2 & 1299.9 & 1310.7 & 1302.2 & 1302.4 & 1299.8 \\
\hline$v 117$ & 1321.2 & 1311.5 & 1313.4 & 1321.6 & 1320.8 & 1302.7 & 1311.0 & 1300.3 \\
\hline$v 118$ & 1329.4 & 1320.7 & 1317.6 & 1324.1 & 1341.4 & 1325.3 & 1345.1 & 1328.6 \\
\hline$v 119$ & 1349.0 & 1331.2 & 1349.0 & 1328.9 & 1359.5 & 1333.5 & 1367.2 & 1339.9 \\
\hline$v 120$ & 1353.0 & 1335.8 & 1353.4 & 1355.7 & 1359.5 & 1362.0 & 1367.2 & 1361.2 \\
\hline$v 121$ & 1361.1 & 1365.0 & 1360.1 & 1368.3 & 1383.8 & 1375.0 & 1389.4 & 1372.9 \\
\hline$v 122$ & 1383.7 & 1383.6 & 1381.7 & 1384.0 & 1383.8 & 1382.9 & 1391.4 & 1384.3 \\
\hline v123 & 1385.3 & 1394.6 & 1384.9 & 1384.3 & 1386.8 & 1398.8 & 1391.4 & 1417.5 \\
\hline v124 & 1395.2 & 1399.0 & 1417.1 & 1390.0 & 1394.0 & 1403.0 & 1412.3 & 1420.0 \\
\hline$v 125$ & 1398.0 & 1406.1 & 1417.3 & 1410.4 & 1436.8 & 1419.2 & 1420.4 & 1423.7 \\
\hline$v 126$ & 1434.4 & 1433.9 & 1418.8 & 1414.4 & 1440.0 & 1431.9 & 1438.2 & 1435.2 \\
\hline$v 127$ & 1438.4 & 1434.8 & 1437.9 & 1438.6 & 1440.0 & 1436.3 & 1438.2 & 1435.2 \\
\hline v128 & 1458.7 & 1436.5 & 1450.4 & 1451.2 & 1453.3 & 1440.0 & 1448.0 & 1438.2 \\
\hline$v 129$ & 1465.0 & 1452.9 & 1458.5 & 1460.3 & 1472.1 & 1445.2 & 1452.5 & 1441.5 \\
\hline$v 130$ & 1475.3 & 1467.0 & 1467.8 & 1474.4 & 1476.4 & 1467.4 & 1471.8 & 1446.5 \\
\hline$v 131$ & 1477.3 & 1477.9 & 1482.7 & 1476.9 & 1497.2 & 1473.6 & 1478.7 & 1480.2 \\
\hline$v 132$ & 1494.0 & 1485.9 & 1488.1 & 1496.0 & 1497.2 & 1484.4 & 1481.7 & 1487.4 \\
\hline$v 133$ & 1500.7 & 1490.6 & 1492.5 & 1501.3 & 1501.7 & 1484.6 & 1481.7 & 1488.9 \\
\hline$v 134$ & 1503.4 & 1493.2 & 1493.9 & 1507.2 & 1507.8 & 1493.8 & 1496.3 & 1489.2 \\
\hline$v 135$ & 1510.7 & 1504.2 & 1502.9 & 1510.3 & 1510.5 & 1494.6 & 1497.7 & 1491.3 \\
\hline$v 136$ & 1512.4 & 1506.9 & 1503.2 & 1510.6 & 1510.5 & 1501.8 & 1497.7 & 1493.2 \\
\hline$v 137$ & 1549.2 & 1524.1 & 1538.2 & 1526.7 & 1511.4 & 1503.4 & 1498.3 & 1509.0 \\
\hline v138 & 1554.0 & 1527.8 & 1552.4 & 1549.7 & 1531.2 & 1505.3 & 1532.4 & 1533.7 \\
\hline v139 & 1558.0 & 1540.3 & 1557.0 & 1555.8 & 1531.2 & 1525.0 & 1532.4 & 1581.2 \\
\hline$v 140$ & 1586.9 & 1550.1 & 1564.8 & 1562.6 & 1545.3 & 1533.5 & 1546.5 & 1593.1 \\
\hline$v 141$ & 1587.7 & 1554.5 & 1565.2 & 1575.7 & 1581.7 & 1579.8 & 1591.7 & 1602.8 \\
\hline$v 142$ & 1609.6 & 1597.8 & 1626.8 & 1599.7 & 1630.3 & 1619.5 & 1640.5 & 1612.8 \\
\hline$v 143$ & 1626.3 & 1614.6 & 1638.0 & 1629.4 & 1631.0 & 1620.0 & 1641.3 & 1625.5 \\
\hline$v 144$ & 1627.2 & 1615.4 & 1638.7 & 1629.9 & 1631.0 & 1635.6 & 1641.3 & 1635.2 \\
\hline$v 145$ & 1642.9 & 1638.3 & 1639.2 & 1636.5 & 1633.1 & 1635.6 & 1642.0 & 1638.2 \\
\hline$v 146$ & 1643.6 & 1639.4 & 1639.6 & 1639.2 & 1655.9 & 1636.8 & 1643.1 & 1638.7 \\
\hline v147 & 1654.9 & 1640.5 & 1648.3 & 1646.9 & 1657.4 & 1637.1 & 1643.1 & 1648.6 \\
\hline$v 148$ & 1655.2 & 1642.2 & 1648.5 & 1652.2 & 1657.4 & 1641.8 & 1643.2 & 1649.7 \\
\hline v149 & 1663.0 & 1646.3 & 1653.8 & 1655.5 & 1658.9 & 1643.5 & 1644.4 & 1655.6 \\
\hline$v 150$ & 1663.2 & 1647.5 & 1654.2 & 1658.6 & 3175.9 & 3174.3 & 3192.4 & 3193.0 \\
\hline$v 151$ & 3174.3 & 3176.6 & 3191.5 & 3189.0 & 3175.9 & 3174.4 & 3192.4 & 3193.0 \\
\hline$v 152$ & 3174.3 & 3176.7 & 3191.5 & 3191.4 & 3175.9 & 3178.1 & 3192.4 & 3193.2 \\
\hline$v 153$ & 3178.6 & 3177.0 & 3194.3 & 3191.4 & 3175.9 & 3178.2 & 3192.4 & 3193.2 \\
\hline$v 154$ & 3178.6 & 3177.1 & 3194.3 & 3201.5 & 3190.3 & 3188.5 & 3204.4 & 3204.8 \\
\hline$v 155$ & 3189.0 & 3191.2 & 3203.7 & 3201.8 & 3190.3 & 3188.5 & 3204.4 & 3204.8 \\
\hline v156 & 3189.0 & 3191.2 & 3203.7 & 3203.7 & 3190.3 & 3192.6 & 3204.4 & 3205.1 \\
\hline$v 157$ & 3193.0 & 3191.3 & 3206.3 & 3203.7 & 3190.4 & 3192.6 & 3204.5 & 3205.1 \\
\hline v158 & 3193.0 & 3191.4 & 3206.3 & 3211.9 & 3205.5 & 3202.6 & 3215.2 & 3214.5 \\
\hline$v 159$ & 3203.9 & 3205.4 & 3214.3 & 3213.0 & 3205.6 & 3202.6 & 3215.3 & 3214.6 \\
\hline$v 160$ & 3203.9 & 3205.4 & 3214.3 & 3215.8 & 3205.6 & 3206.4 & 3215.3 & 3215.4 \\
\hline v161 & 3207.4 & 3206.2 & 3217.9 & 3215.8 & 3205.7 & 3206.5 & 3215.5 & 3215.4 \\
\hline$v 162$ & 3207.5 & 3206.3 & 3218.0 & 3216.6 & 3208.8 & 3206.7 & 3218.7 & 3218.5 \\
\hline$v 163$ & 3209.2 & 3208.8 & 3218.2 & 3219.5 & 3208.9 & 3206.7 & 3218.8 & 3218.5 \\
\hline v164 & 3209.2 & 3208.9 & 3218.2 & 3219.5 & 3208.9 & 3210.0 & 3218.8 & 3218.9 \\
\hline$v 165$ & 3212.1 & 3209.6 & 3221.2 & 3230.4 & 3209.1 & 3210.1 & 3218.9 & 3219.0 \\
\hline v166 & 3212.2 & 3209.7 & 3221.2 & 3231.7 & & & & \\
\hline v167 & 3524.8 & 3520.3 & 3526.3 & 3547.8 & & & & \\
\hline$v 168$ & 3586.7 & 3581.0 & 3590.8 & 3606.6 & & & & \\
\hline
\end{tabular}


(a) $\mathrm{H}_{2} \mathrm{PC}$

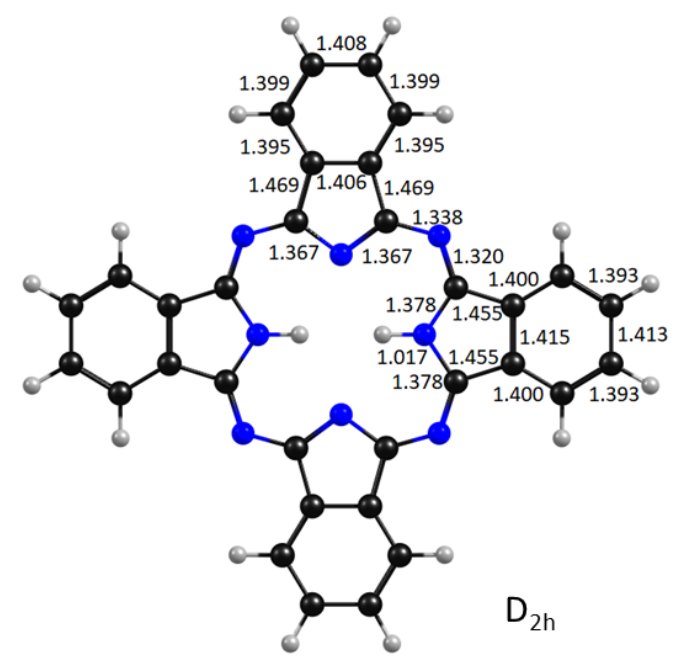

(c) $\mathrm{ZnPc}$

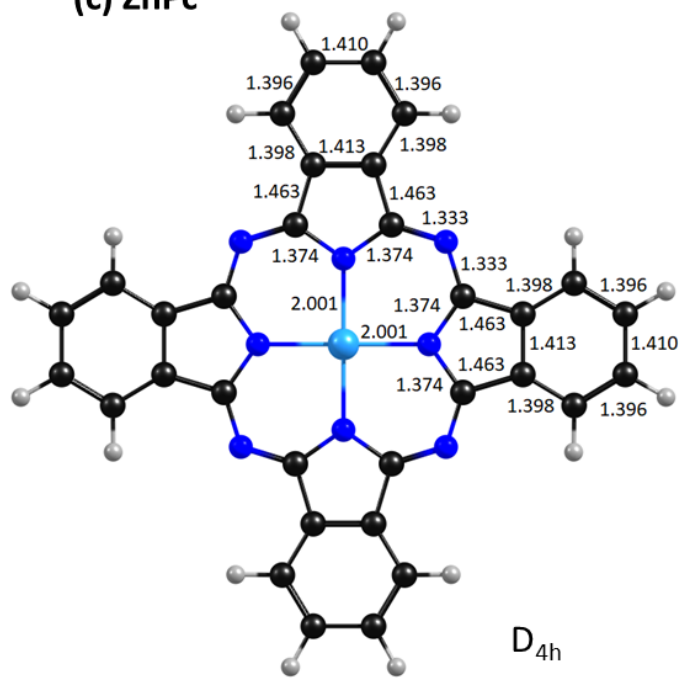

(b) $\mathrm{H}_{2} \mathrm{Pc}^{+}$

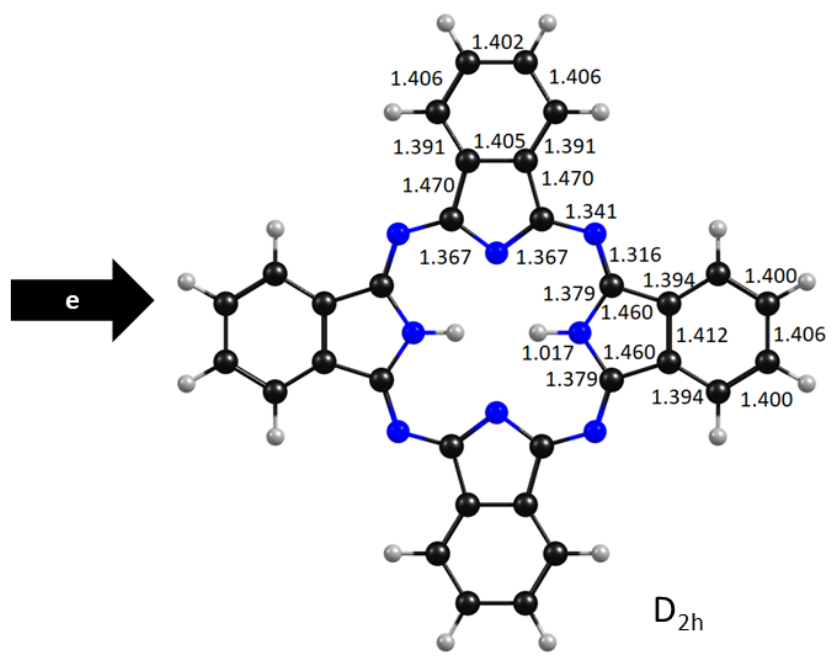

(d) $\mathrm{ZnPc}^{+}$

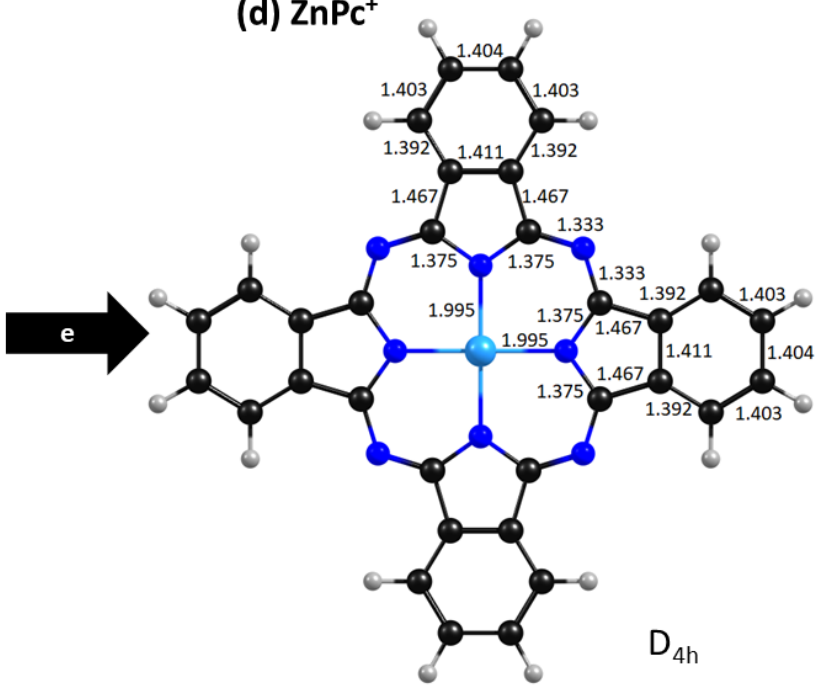

Figure S1. Optimized geometries of ground states of $\mathrm{H}_{2} \mathrm{Pc}(\mathrm{a}), \mathrm{H}_{2} \mathrm{Pc}^{+}(\mathrm{b}), \mathrm{ZnPc}(\mathrm{c})$, and $\mathrm{ZnPc} \mathrm{C}^{+}(\mathrm{d})$ calculated with the B3LYP/cc-pVDZ method; bond lengths are given in $\AA$. 


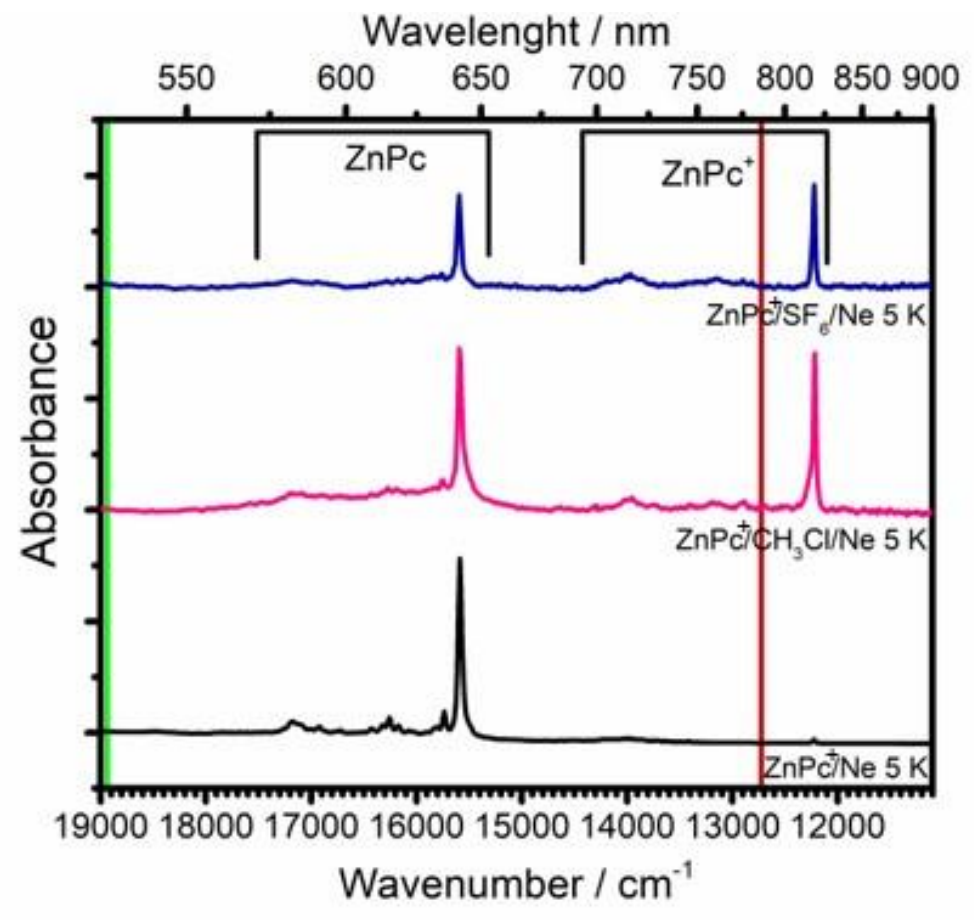

Figure S2. Absorption spectra recorded for $\mathrm{ZnPc}^{+} / \mathrm{Ne}, \mathrm{ZnPc}^{+} / \mathrm{CH}_{3} \mathrm{Cl} / \mathrm{Ne}$, and $\mathrm{ZnPc}+\mathrm{SF}_{6} / \mathrm{Ne}$ at $5 \mathrm{~K}$. 


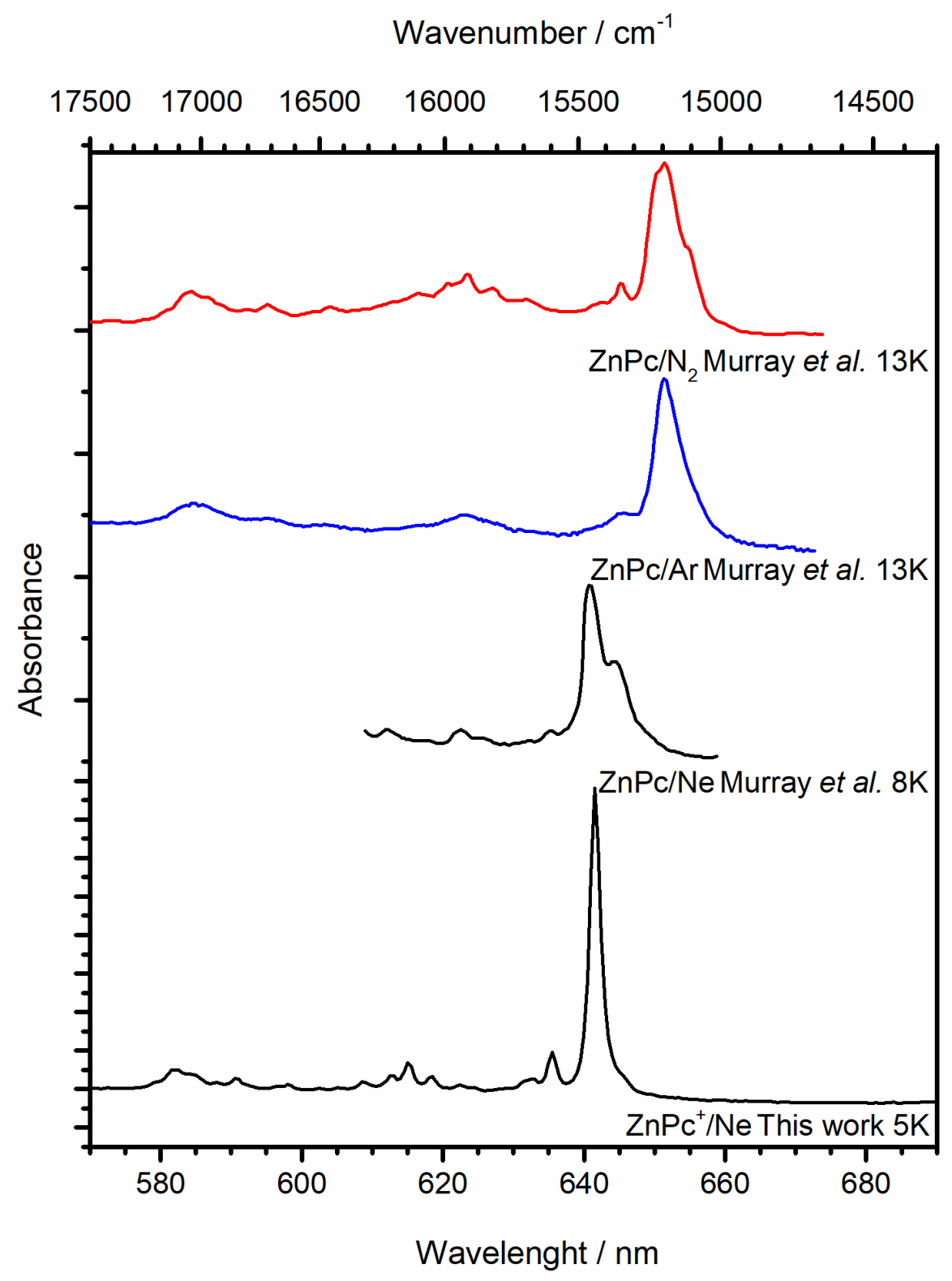

Figure S3. Comparison of the absorption spectrum of ZnPc recorded in this work with previous studies in $\mathrm{Ne}, \mathrm{Ar}$, and $\mathrm{N}_{2}$ matrices; The spectra in $\mathrm{Ne}, \mathrm{Ar}$, and $\mathrm{N}_{2}$ matrices were produced from the digitized data extracted from Fig. 2 of ref. 24. 
Table S2. Band maxima ( $\pm 1 \mathrm{~nm}$ ) of the $\mathrm{S}_{0} \rightarrow \mathrm{S}_{1}$ absorption of $\mathrm{ZnPc}$, isolated in solid $\mathrm{Ne}$ at $5 \mathrm{~K}$, compared with literature data acquired in other matrix isolation experiments and in gas-phase.

\begin{tabular}{|c|c|c|c|c|c|c|c|c|}
\hline \multicolumn{3}{|c|}{ This work } & \multicolumn{5}{|c|}{ Murray et al. } & \multirow{3}{*}{$\begin{array}{c}\text { Plows and Jones } \\
\text { Gas-phase } \\
\Delta \mathbf{v}^{\mathrm{b}} \\
/ \mathrm{cm}^{-1}\end{array}$} \\
\hline \multicolumn{3}{|c|}{ Experimental $\mathrm{Ne}$} & \multirow{2}{*}{$\begin{array}{c}\mathrm{N}_{2} \\
\Delta v^{\mathrm{b}} \\
/ \mathrm{cm}^{-1}\end{array}$} & \multirow{2}{*}{$\begin{array}{c}A r \\
\Delta v^{\mathrm{b}} \\
/ \mathrm{cm}^{-1}\end{array}$} & \multirow{2}{*}{$\begin{array}{c}\mathrm{Kr} \\
\Delta v^{\mathrm{b}} \\
/ \mathrm{cm}^{-1}\end{array}$} & \multirow{2}{*}{$\begin{array}{c}\mathrm{Xe} \\
\Delta \mathrm{v}^{\mathrm{b}} \\
/ \mathrm{cm}^{-1}\end{array}$} & \multirow[b]{2}{*}{ Symmetry } & \\
\hline $\begin{array}{c}\lambda_{\mathrm{Ne}} \\
/ \mathrm{nm}\end{array}$ & $\begin{array}{c}v \\
/ \mathrm{cm}^{-1} \mathrm{a}\end{array}$ & $\begin{array}{c}\Delta v^{b} \\
/ \mathrm{cm}^{-1}\end{array}$ & & & & & & \\
\hline \multirow[t]{2}{*}{641} & 15589 & 0 & & & & & & \\
\hline & & & & 87 & & & $\mathrm{~B}_{2 \mathrm{~g}}$ & 103 \\
\hline 635 & 15736 & 147 & 164 & 154 & 155 & 155 & $B_{1 g}$ & 153 \\
\hline 632 & 15810 & 221 & 229 & 225 & 224 & 227 & $\mathrm{~B}_{2 \mathrm{~g}}$ & 226 \\
\hline 631 & 15835 & 246 & 259 & 252 & 253 & 255 & $A_{1 g}$ & 256 \\
\hline \multirow[t]{3}{*}{629} & 15898 & 309 & & 314 & 315 & 314 & $\mathrm{E}_{\mathrm{g}}$ & 306 \\
\hline & & & & 356 & & & & 324 \\
\hline & & & & 412 & 410 & 410 & $\mathrm{Eg}_{\mathrm{g}}$ & 419 \\
\hline 624 & 16012 & 424 & & 439 & & & & 430 \\
\hline 622 & 16064 & 475 & 479 & 478 & 476 & 479 & $\mathrm{~B}_{2 \mathrm{~g}}$ & 482 \\
\hline 618 & 16168 & 579 & 593 & 586 & 487 & 586 & $A_{1 g}$ & 565 \\
\hline 615 & 16259 & 671 & 676 & 675 & 675 & 671 & $A_{1 g}$ & 589 \\
\hline \multirow[t]{2}{*}{612} & 16326 & 737 & 744 & 742 & 742 & 737 & $\mathrm{~B}_{1 \mathrm{~g}}$ & \\
\hline & & & 832 & 837 & 838 & 837 & & \\
\hline 608 & 16433 & 844 & 846 & 851 & & & & \\
\hline 605 & 16528 & 939 & & 940 & 942 & & $\mathrm{~B}_{2 \mathrm{~g}}$ & \\
\hline 602 & 16596 & 1007 & & & & & & \\
\hline 598 & 16720 & 1132 & & & & & & \\
\hline 596 & 16763 & 1174 & & & & & & \\
\hline 590 & 16933 & 1344 & & & & & & \\
\hline 588 & 17005 & 1416 & & & & & & \\
\hline 585 & 17106 & 1517 & & & & & & \\
\hline 584 & 17121 & 1532 & & & & & & \\
\hline 582 & 17180 & 1591 & & & & & & \\
\hline 577 & 17343 & 1755 & & & & & & \\
\hline 572 & 17464 & 1875 & & & & & & \\
\hline
\end{tabular}


Table S3. Observed band maxima in the absorption spectra of $\mathrm{H}_{2} \mathrm{Pc}$ isolated in solid $\mathrm{Ne}$ at $5 \mathrm{~K}$.

\begin{tabular}{|c|c|c|}
\hline$\lambda_{\mathrm{Ne}} / \mathrm{nm}$ & $\mathrm{v} / \mathrm{cm}^{-1 \mathrm{a}}$ & $\Delta v^{b} / \mathrm{cm}^{-1}$ \\
\hline 666.4 & 15005 & 0 \\
\hline 660.9 & 15131 & 126 \\
\hline 656.9 & 15223 & 218 \\
\hline 655.1 & 15265 & 260 \\
\hline 652.1 & 15336 & 330 \\
\hline 645.2 & 15499 & 494 \\
\hline 642.1 & 15575 & 570 \\
\hline 637.9 & 15678 & 672 \\
\hline 633.2 & 15792 & 786 \\
\hline 630.0 & 15873 & 868 \\
\hline 627.6 & 15934 & 929 \\
\hline 623.2 & 16046 & 1041 \\
\hline 620.1 & 16126 & 1121 \\
\hline 614.2 & 16282 & 1277 \\
\hline 614.2 & 16282 & 1277 \\
\hline 614.2 & 16282 & 1277 \\
\hline 611.3 & 16358 & 1353 \\
\hline 607.7 & 16455 & 1449 \\
\hline 605.2 & 16523 & 1518 \\
\hline 601.9 & 16613 & 1608 \\
\hline 596.5 & 16764 & 1759 \\
\hline 593.6 & 16845 & 1840 \\
\hline 587.5 & 17022 & 2017 \\
\hline 581.2 & 17206 & 2201 \\
\hline 573.5 & 17436 & 2431 \\
\hline 566.2 & 17662 & 2657 \\
\hline 561.0 & 17826 & 2820 \\
\hline
\end{tabular}

${ }^{\mathrm{a}} v=1 / \lambda_{\mathrm{Ne}}$

${ }^{\mathrm{b}}$ Wavenumber difference with respect to the 0-0 transition

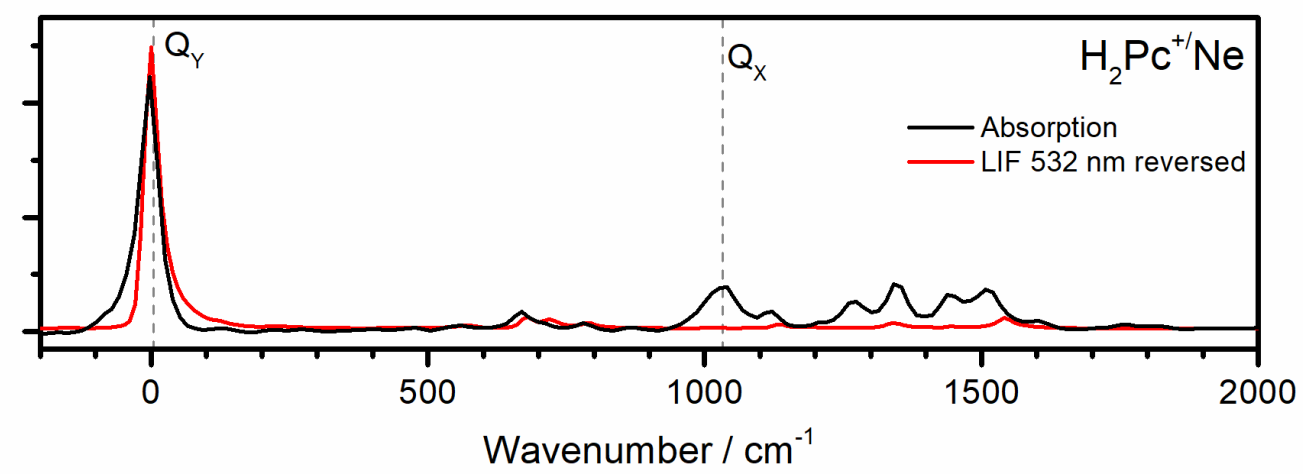

Figure S4. Comparison of absorption and reversed emission (532 $\mathrm{nm}$ excitation) spectra of $\mathrm{H}_{2} \mathrm{Pc}$ isolated in solid Ne. 
Table S4. Vertical excitation wavelengths and oscillator strengths of $\mathrm{ZnPc}, \mathrm{ZnPc}^{+}, \mathrm{H}_{2} \mathrm{Pc}$, and $\mathrm{H}_{2} \mathrm{Pc}^{+}$electronic transitions calculated with the TD-B3LYP/cc-pVDZ method.

\begin{tabular}{|c|c|c|c|}
\hline \multicolumn{2}{|c|}{$\mathrm{ZnPc}$} & \multicolumn{2}{|c|}{$\mathrm{ZnPc}^{+}$} \\
\hline Wavelength $\lambda / \mathrm{nm}$ & Oscillator strength f & Wavelength $\lambda / \mathrm{nm}$ & Oscillator strength $\mathrm{f}$ \\
\hline 595.31 & 0.4251 & 758.33 & 0.0598 \\
\hline 595.31 & 0.4251 & 758.33 & 0.0598 \\
\hline 390.37 & 0 & 684.13 & 0 \\
\hline 390.37 & 0 & 666.57 & 0.0013 \\
\hline 370.66 & 0 & 666.57 & 0.0013 \\
\hline 370.49 & 0 & 646.10 & 0 \\
\hline 367.39 & 0.0087 & 620.01 & 0 \\
\hline 367.39 & 0.0087 & 595.75 & 0 \\
\hline 360.61 & 0 & 577.49 & 0.3146 \\
\hline 360.61 & 0 & 577.49 & 0.3146 \\
\hline 360.09 & 0 & 563.16 & 0 \\
\hline 354.79 & 0 & 546.61 & 0 \\
\hline 348.54 & 0 & 485.65 & 0 \\
\hline 345.63 & 0 & 466.66 & 0 \\
\hline 340.05 & 0 & 466.66 & 0 \\
\hline 338.09 & 0.1779 & 432.24 & 0.0153 \\
\hline 338.09 & 0.1779 & 432.24 & 0.0153 \\
\hline 337.37 & 0 & 426.57 & 0 \\
\hline 331.40 & 0.3133 & 424.34 & 0 \\
\hline 331.40 & 0.3133 & 404.50 & 0 \\
\hline \multicolumn{2}{|c|}{$\mathrm{H}_{2} \mathrm{Pc}$} & \multicolumn{2}{|c|}{$\mathrm{H}_{2} \mathrm{Pc}^{+}$} \\
\hline Wavelength $\lambda / \mathrm{nm}$ & Oscillator strength f & Wavelength $\lambda / \mathrm{nm}$ & Oscillator strength $f$ \\
\hline 601.60 & 0.3941 & 799.09 & 0.0735 \\
\hline 595.39 & 0.445 & 770.09 & 0.0105 \\
\hline 404.17 & 0.0678 & 762.77 & 0 \\
\hline 402.82 & 0 & 719.88 & 0.0527 \\
\hline 377.31 & 0.0059 & 636.14 & 0 \\
\hline 375.36 & 0 & 606.76 & 0.3144 \\
\hline 358.29 & 0 & 603.31 & 0 \\
\hline 354.52 & 0 & 573.06 & 0.0094 \\
\hline 352.61 & 0 & 554.24 & 0.2805 \\
\hline 350.79 & 0 & 538.44 & 0 \\
\hline 345.01 & 0 & 533.32 & 0 \\
\hline 338.87 & 0 & 481.16 & 0 \\
\hline 338.61 & 0.2254 & 479.69 & 0 \\
\hline 337.63 & 0.1671 & 474.15 & 0.0135 \\
\hline 334.62 & 0 & 472.20 & 0 \\
\hline 334.03 & 0.1612 & 453.70 & 0.0263 \\
\hline 329.95 & 0 & 452.38 & 0 \\
\hline 329.53 & 0 & 427.53 & 0 \\
\hline 328.07 & 0.8901 & 423.96 & 0 \\
\hline 327.46 & 0.0072 & 420.77 & 0.0948 \\
\hline
\end{tabular}




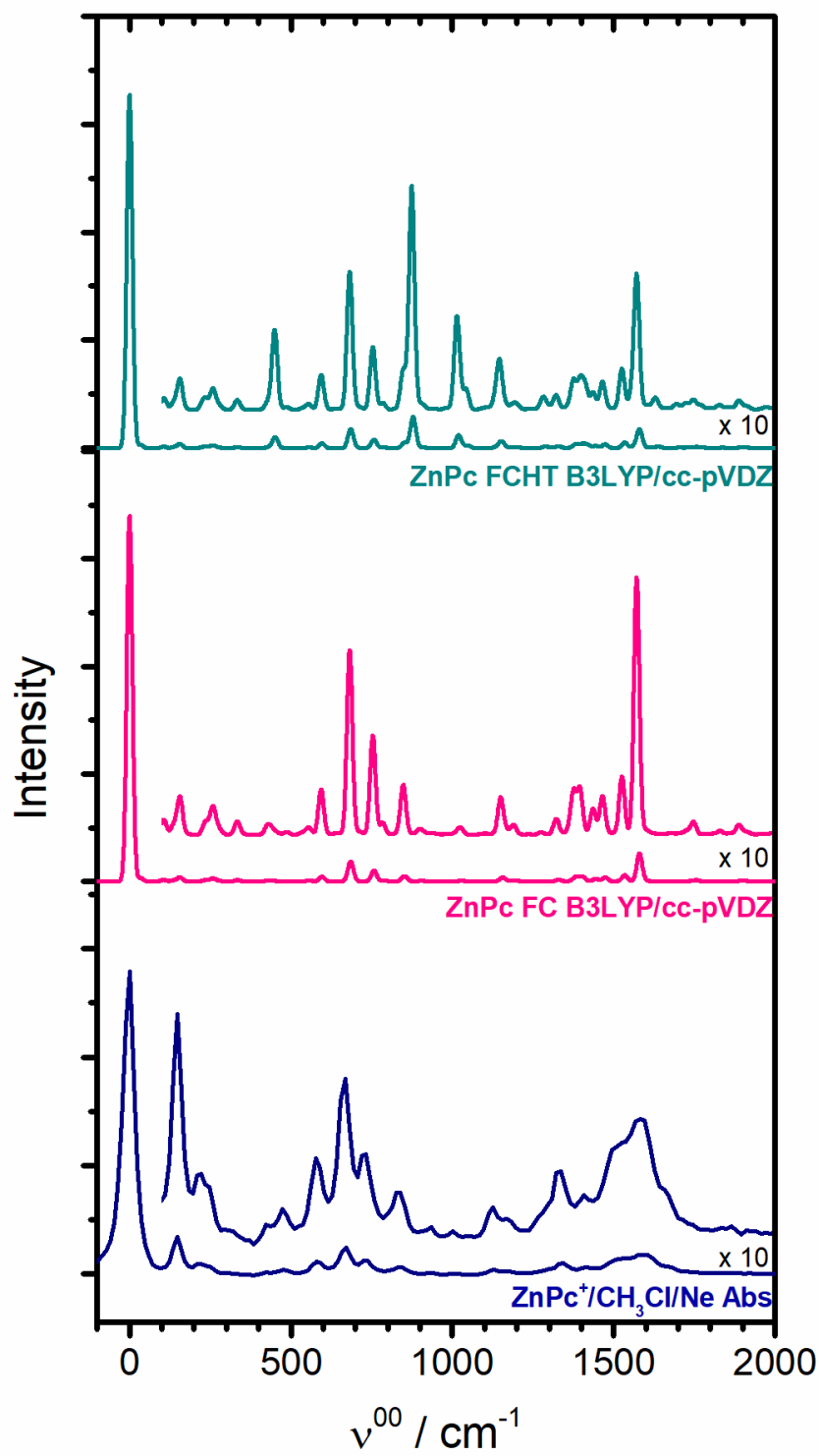

Figure S5. Comparison of the $\mathrm{ZnPc}$ absorption spectrum (recorded in a $\mathrm{ZnPc}+\mathrm{CH}_{3} \mathrm{Cl} / \mathrm{Ne}$ matrix) with predicted spectral pattern on the basis of our FC and FCHT TD-B3LYP/cc-pVDZ calculations 

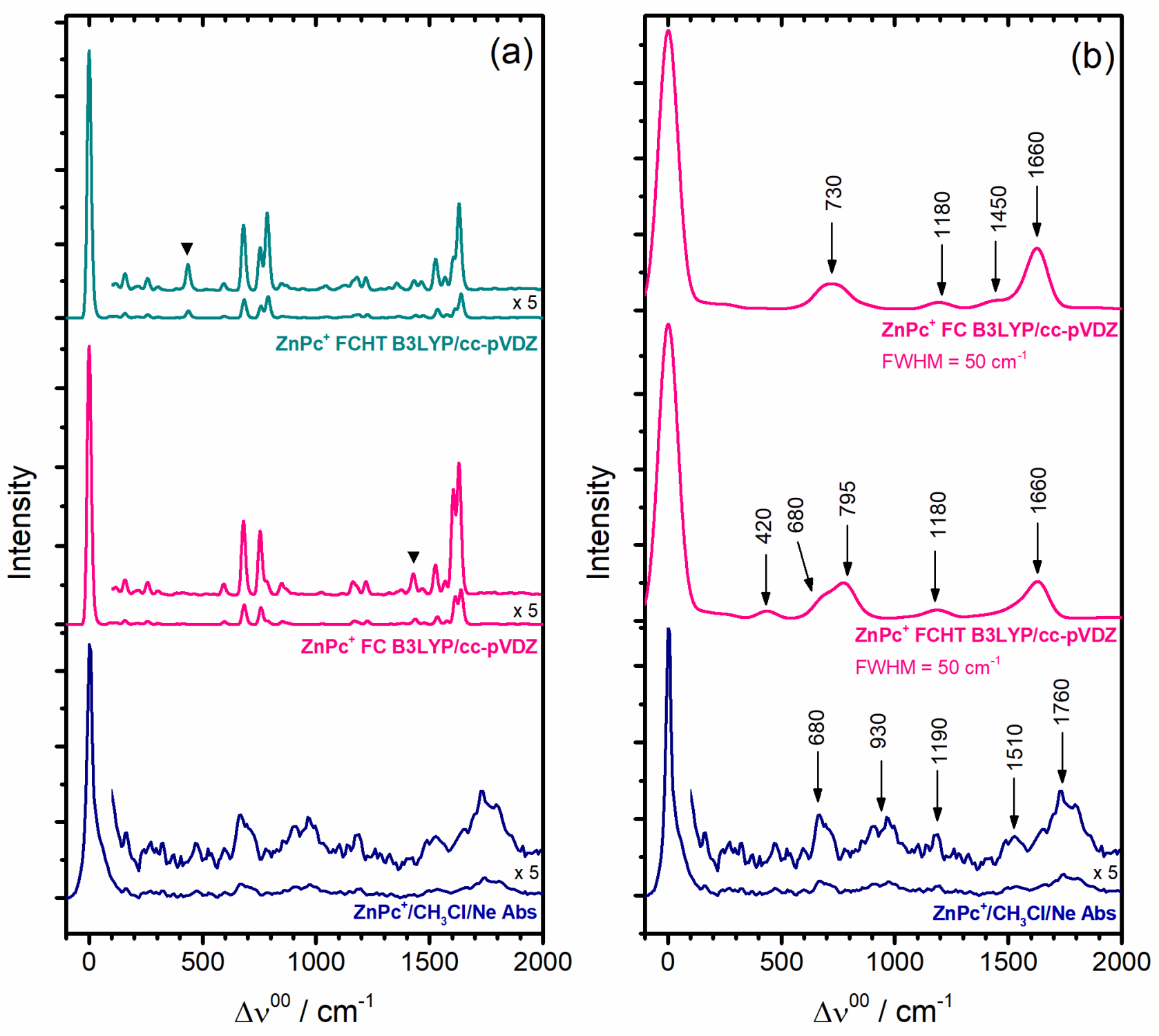

Figure S6. Comparison of the vibrational pattern associated with the $\mathrm{D}_{0} \rightarrow \mathrm{D}_{1}$ transition of $\mathrm{ZnPc}^{+}$isolated in solid $\mathrm{Ne}\left(0-0\right.$ origin band at $\left.12153 \mathrm{~cm}^{-1}\right)$ with the predicted Franck-Condon (FC) and Franck-Condon Hertzberg-Teller (FCHT) spectra based on TD-B3LYP/cc-pVDZ calculations with FWMH = 10 and $50 \mathrm{~cm}^{-1}$ in (a) and (b), respectively; The vibrational frequencies are plotted relative to the $0-0$ transition; triangles indicate the lines discussed in the main text. 
Table S5. $\mathrm{S}_{1} \rightarrow \mathrm{S}_{0}$ fluorescence of $\mathrm{ZnPc}$ isolated in solid $\mathrm{Ne}$ at $5 \mathrm{~K}$ as measured in this work, compared with literature data acquired in other matrix isolation experiments and in STM-LE measurements.

\begin{tabular}{|c|c|c|c|c|c|c|c|c|c|}
\hline \multicolumn{3}{|c|}{ This work } & \multirow{2}{*}{\multicolumn{2}{|c|}{$\begin{array}{c}\text { Doppagne et al. } \\
\text { STM-LE }\end{array}$}} & \multicolumn{5}{|c|}{ Murray et al. } \\
\hline \multicolumn{3}{|c|}{$\mathrm{Ne}$} & & & \multirow{2}{*}{$\begin{array}{c}\mathrm{Ne} \\
\Delta \mathrm{v}^{\mathrm{b}} \\
/ \mathrm{cm}^{-1}\end{array}$} & \multirow{2}{*}{$\begin{array}{c}\mathrm{N}_{2} \\
\Delta \mathrm{v}^{\mathrm{b}} \\
/ \mathrm{cm}^{-1}\end{array}$} & \multirow{2}{*}{$\begin{array}{c}\mathrm{Ar} \\
\Delta \mathrm{v}^{\mathrm{b}} \\
/ \mathrm{cm}^{-1}\end{array}$} & \multirow{2}{*}{$\begin{array}{c}\mathrm{Kr} \\
\Delta \mathrm{v}^{\mathrm{b}} \\
/ \mathrm{cm}^{-1}\end{array}$} & \multirow{2}{*}{$\begin{array}{c}\mathrm{Xe} \\
\Delta \mathrm{v}^{\mathrm{b}} \\
/ \mathrm{cm}^{-1}\end{array}$} \\
\hline $\begin{array}{l}\lambda_{\mathrm{Ne}} \\
/ \mathrm{nm}\end{array}$ & $\begin{array}{c}\mathrm{v} \\
/ \mathrm{cm}^{-1} \mathrm{a}\end{array}$ & $\begin{array}{c}\Delta \mathrm{v}^{\mathrm{b}} \\
/ \mathrm{cm}^{-1}\end{array}$ & $\begin{array}{c}\Delta \mathrm{v}^{\mathrm{b}} \\
/ \mathrm{cm}^{-1 \mathrm{c}}\end{array}$ & Symm. & & & & & \\
\hline \multirow[t]{2}{*}{641} & 15597 & 0 & & & & & & & \\
\hline & & & & & & -21 & -34 & -79 & \\
\hline 645 & 15510 & -87 & & & & -104 & -102 & -104 & -102 \\
\hline 648 & 15441 & -156 & & & & -163 & -161 & -161 & -161 \\
\hline 651 & 15370 & -227 & & & & -227 & -237 & -237 & \\
\hline \multirow[t]{2}{*}{652} & 15344 & -253 & & & & -262 & -260 & -260 & -265 \\
\hline & & & $\sim 410$ & & & & & & \\
\hline 662 & 15117 & -480 & $\sim 490$ & $B_{2 G}$ & -495 & -488 & -490 & -491 & -488 \\
\hline 666 & 15007 & -590 & $\sim 590$ & $A_{1 G}$ & -595 & -597 & -598 & -598 & \\
\hline 670 & 14919 & -678 & $\sim 680$ & $A_{1 G}$ & -673 & -683 & -689 & -689 & \\
\hline 673 & 14848 & -749 & $\sim 750$ & $\mathrm{~B}_{1 \mathrm{G}}$ & -751 & -752 & -751 & -751 & \\
\hline 677 & 14762 & -835 & $\sim 830$ & $A_{1 G}$ & -841 & -845 & -845 & -845 & \\
\hline 681 & 14694 & -948 & & & -956 & -945 & -945 & -945 & -954 \\
\hline 685 & 14590 & -1007 & & & & & & & \\
\hline 692 & 14456 & -1141 & $\sim 1140$ & $\mathrm{~B}_{1 \mathrm{G}}$ & -1151 & -1147 & -1150 & -1149 & -1152 \\
\hline 695 & 14390 & -1207 & $\sim 1210$ & $B_{2 G}$ & & -1215 & -1221 & -1221 & -1223 \\
\hline 701 & 14260 & -1337 & $\sim 1340$ & $A_{1 G}$ & -1346 & -1346 & -1346 & -1346 & -1350 \\
\hline 707 & 14152 & -1445 & 1440 & $\mathrm{B}_{1 \mathrm{G}}$ & -1451 & -1448 & -1448 & -1448 & -1452 \\
\hline 710 & 14084 & -1513 & 1510 & $\mathrm{B}_{1 \mathrm{G}}$ & -1519 & -1525 & -1525 & -1524 & -1522 \\
\hline 715 & 13993 & -1604 & & & -1614 & & -1624 & -1610 & -1613 \\
\hline 718 & 13929 & -1668 & & & & & & & \\
\hline 750 & 13334 & -2263 & & & & & & & \\
\hline
\end{tabular}

${ }^{\mathrm{a}} \mathrm{v}=1 / \lambda_{\mathrm{Ne}}$

${ }^{\mathrm{b}}$ Wavenumber difference in respect to the 0-0 transition

${ }^{d}$ Estimated from Figure 2 of reference ${ }^{4}$ 
Table S6. $\mathrm{S}_{1} \rightarrow \mathrm{S}_{0}$ fluorescence of $\mathrm{H}_{2} \mathrm{Pc}$ isolated in solid $\mathrm{Ne}$ at $5 \mathrm{~K}$, compared with literature data acquired in other matrix isolation experiments and in gas-phase; wavenumbers in respect to 0-0 band origin at 14984 $\mathrm{cm}^{-1}$.

\begin{tabular}{|c|c|c|c|c|c|c|c|}
\hline \multirow{2}{*}{$\begin{array}{c}\text { This work } \\
\mathrm{Ne}\end{array}$} & \multicolumn{7}{|c|}{ Murray at al. } \\
\hline & $\mathrm{Ne}$ & $\mathbf{N}_{2}$ & $\mathrm{Ar}$ & $\mathrm{Kr}$ & $\mathrm{Xe}$ & Shpol'skii & Gas phase \\
\hline \multicolumn{8}{|l|}{-64} \\
\hline \multicolumn{8}{|l|}{-119} \\
\hline & & & -140 & -143 & & -36 & -132 \\
\hline & & & -190 & & & -184 & -184 \\
\hline \multicolumn{8}{|l|}{-217} \\
\hline & & & -238 & -238 & & -232 & -231 \\
\hline \multicolumn{8}{|l|}{-336} \\
\hline & & & -489 & -494 & & -487 & -484 \\
\hline & & & & -544 & & -542 & -544 \\
\hline-568 & & -574 & -576 & -576 & -572 & -569 & -574 \\
\hline-682 & -688 & -687 & -688 & -688 & -682 & -683 & -685 \\
\hline \multirow[t]{2}{*}{-722} & -726 & -731 & -730 & -731 & -727 & -725 & -729 \\
\hline & & & & -771 & & -769 & -768 \\
\hline \multirow[t]{4}{*}{-793} & & -802 & -802 & -802 & -800 & -801 & -799 \\
\hline & & & & & -965 & -1009 & -1012 \\
\hline & & & & -1031 & -1030 & -1030 & -1037 \\
\hline & -1070 & & & & -1090 & -1107 & -1111 \\
\hline-1137 & -1148 & & -1145 & -1145 & -1143 & -1141 & -1144 \\
\hline \multirow[t]{2}{*}{-1184} & & & -1188 & -1190 & -1184 & $-1183 / 1188$ & -1189 \\
\hline & & & & & -1253 & -1233 & -1233 \\
\hline \multirow[t]{2}{*}{-1344} & & -1356 & -1350 & -1351 & -1350 & -1352 & -1346 \\
\hline & -1383 & & & & -1376 & -1372 & -1375 \\
\hline \multirow[t]{3}{*}{1446} & -1472 & -1460 & -1456 & -1456 & -1456 & -1453 & -1456 \\
\hline & & & -1523 & & & -1522 & -1517 \\
\hline & & & & & & $1542 / 1546$ & -1535 \\
\hline-1546 & -1547 & -1553 & -1550 & -1550 & -1554 & -1555 & -1545 \\
\hline-1617 & & & & & & & \\
\hline
\end{tabular}




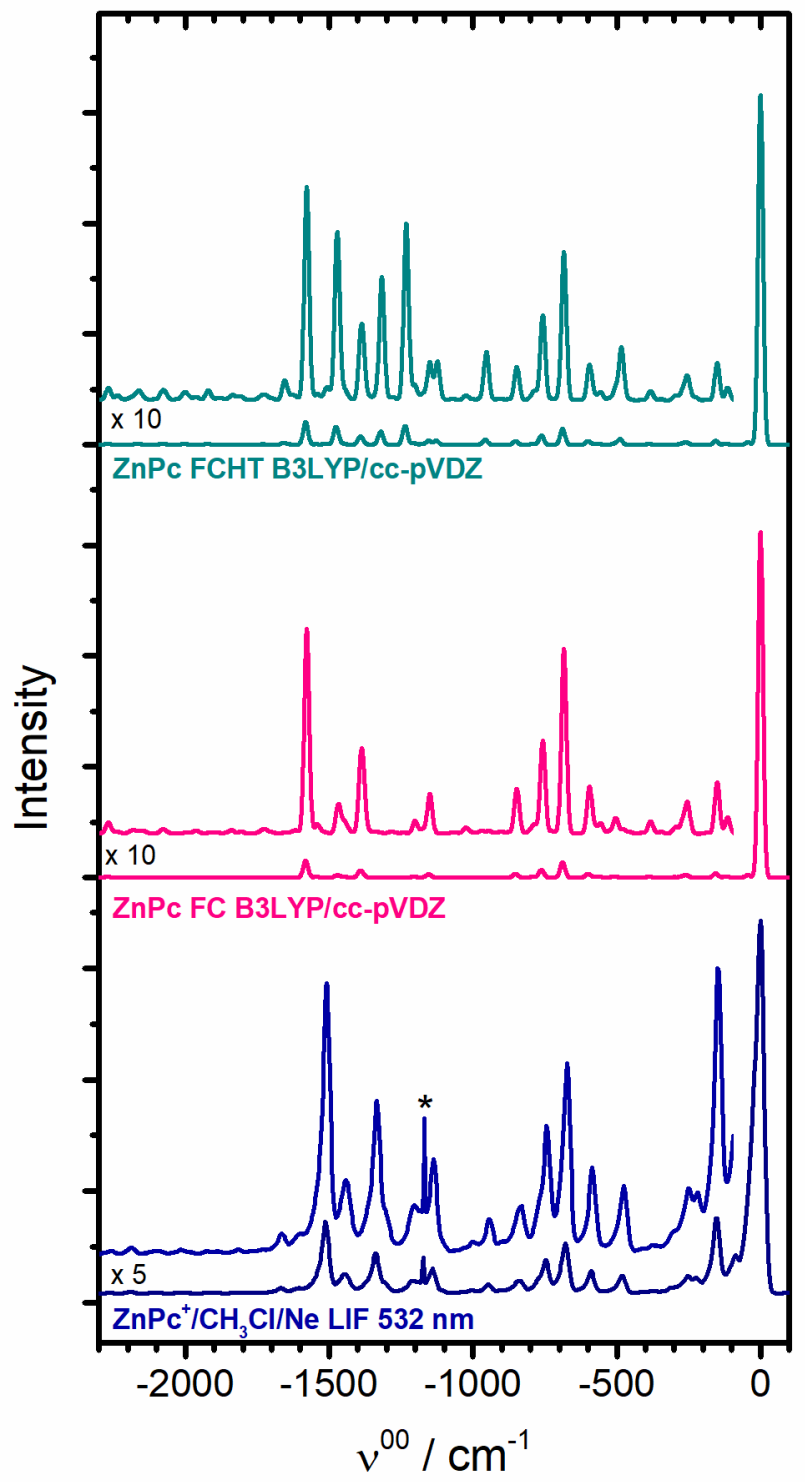

Figure S7. Comparison of the $\mathrm{ZnPc}$ LIF spectrum recorded in this work (in a $\mathrm{ZnPc} / \mathrm{CH}_{3} \mathrm{Cl} / \mathrm{Ne}$ matrix) with the predicted spectral pattern on the basis of our FC and FCHT TD-B3LYP/cc-pVDZ calculations. 


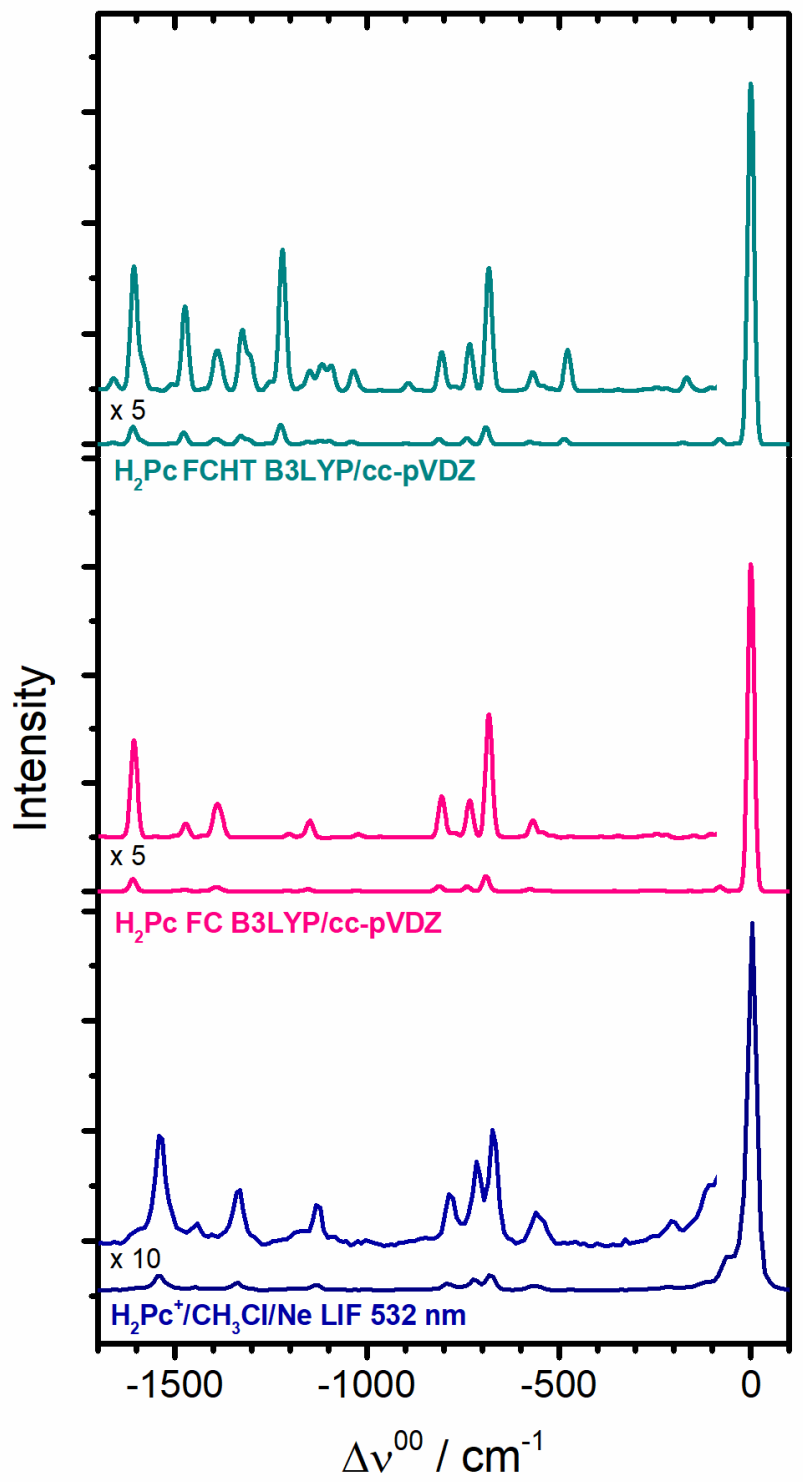

Figure S8. Comparison of the $\mathrm{H}_{2} \mathrm{Pc}$ LIF spectrum recorded in this work (in a $\mathrm{H}_{2} \mathrm{Pc}^{+} / \mathrm{CH}_{3} \mathrm{Cl} / \mathrm{Ne}$ matrix) with predicted spectral pattern on the basis of our FC and FCHT TD-B3LYP/cc-pVDZ calculations. 
Table S7. $\mathrm{S}_{1} \rightarrow \mathrm{S}_{0}$ fluorescence of $\mathrm{ZnPc}^{+}$. Comparison of vibrational features observed in the LIF spectra of matrix isolated $\mathrm{ZnPc}^{+}$with (i) those acquired in STM-LE measurements and (ii) predictions of TD-B3LYP/ccpVDZ calculations at the FC and FCHT levels (vibrotational assignments (final states mostly correspond to single excitations of fundamental modes)), excitation energies (in $\mathrm{cm}^{-1}$ ) and emission intensities).

\begin{tabular}{|c|c|c|c|c|c|c|c|c|}
\hline \multirow{2}{*}{\multicolumn{3}{|c|}{$\begin{array}{c}\text { This work } \\
\mathrm{Ne}\end{array}$}} & \multirow{3}{*}{$\begin{array}{c}\text { Doppagne et al. } \\
\text { STM-LE } \\
\Delta v \\
/ \mathrm{cm}^{-1}\end{array}$} & \multicolumn{4}{|c|}{ TD-B3LYP/cc-pVDZ } & \multirow[b]{3}{*}{ Assignment } \\
\hline & & & & \multicolumn{2}{|r|}{ FC } & \multicolumn{2}{|c|}{ FCHT } & \\
\hline $\begin{array}{c}\lambda_{\mathrm{Ne}} \\
/ \mathrm{nm}\end{array}$ & $\begin{array}{c}\mathrm{v} \\
/ \mathrm{cm}^{-1} \mathrm{a}\end{array}$ & $\begin{array}{c}\Delta v \\
/ \mathrm{cm}^{-1 b}\end{array}$ & & $\begin{array}{c}\Delta v \\
/ \mathrm{cm}^{-1}\end{array}$ & $\begin{array}{c}\text { Int. } \\
/ \mu \mathrm{Jol}^{-1}\end{array}$ & $\begin{array}{c}\Delta v \\
/ \mathrm{cm}^{-1}\end{array}$ & $\begin{array}{c}\text { Int. } \\
/ \mu \mathrm{J} \mathrm{mol}^{-1}\end{array}$ & \\
\hline 938.1 & 10660 & -1562 & & -1628 & 18 & -1628 & 25 & $\left.|0>\rightarrow| 141^{1} ; 1^{2}\right\rangle$ \\
\hline \multirow[t]{2}{*}{936.3} & 10680 & -1542 & $\sim 1550$ & -1592 & 231 & -1592 & 319 & $\left.|0>\rightarrow| 141^{1}\right\rangle$ \\
\hline & & & & -1479 & 4 & -1479 & 7 & $\left.|0>\rightarrow| 131^{1}\right\rangle$ \\
\hline \multirow[t]{4}{*}{926.3} & 10796 & -1426 & $\sim 1410$ & & & -1472 & 34 & $|0>\rightarrow| 130^{1}>$ \\
\hline & & & & & & -1453 & 4 & $|0>\rightarrow| 129^{1}>$ \\
\hline & & & & -1446 & 4 & -1446 & 5 & $\left.|0>\rightarrow| 59^{1} ; 52^{1}\right\rangle$ \\
\hline & & & & & & -1420 & 3 & $|0>\rightarrow| 125^{1}>$ \\
\hline 921.8 & 10848 & -1374 & & -1412 & 9 & -1412 & 15 & $\left.|0>\rightarrow| 124^{1}\right\rangle$ \\
\hline 918.5 & 10887 & -1335 & & -1389 & 8 & -1389 & 15 & $\left.|0>\rightarrow| 121^{1}\right\rangle$ \\
\hline \multirow[t]{2}{*}{915.2} & 10927 & -1295 & & & & -1311 & 20 & $|0>\rightarrow| 117^{1}>$ \\
\hline & & & & & & -1259 & 6 & $\left.|0>\rightarrow| 113^{1} ; 1^{2}\right\rangle$ \\
\hline \multirow[t]{2}{*}{907.3} & 11022 & -1200 & $\sim 1190$ & & & -1223 & 73 & $\left.|0>\rightarrow| 113^{1}\right\rangle$ \\
\hline & & & & -1198 & 12 & -1198 & 6 & $|0>\rightarrow| 110^{1}>$ \\
\hline 898.6 & 11128 & -1094 & 1090 & -1166 & 16 & -1166 & 18 & $|0>\rightarrow| 105^{1}>$ \\
\hline 895.8 & 11163 & -1059 & & & & -1113 & 34 & $|0>\rightarrow| 100^{1}>$ \\
\hline 893.0 & 11198 & -1024 & & & & -1049 & 10 & $\left.|0>\rightarrow| 94^{1}\right\rangle$ \\
\hline 886.3 & 11283 & -939 & & & & -957 & 5 & $\left.|0>\rightarrow| 81^{1}\right\rangle$ \\
\hline \multirow[t]{2}{*}{878.5} & 11383 & -839 & $\sim 830$ & -853 & 12 & -853 & 15 & $\left.|0>\rightarrow| 73^{1}\right\rangle$ \\
\hline & & & & -797 & 7 & -797 & 7 & $\left.|0>\rightarrow| 59^{1} ; 1^{2}\right\rangle$ \\
\hline 872.4 & 11463 & -759 & & -788 & 12 & -788 & 12 & $\left.|0>\rightarrow| 62^{1}\right\rangle$ \\
\hline \multirow[t]{2}{*}{871.0} & 11481 & -741 & $\sim 740$ & -761 & 83 & -761 & 93 & $\left.|0>\rightarrow| 59^{1}\right\rangle$ \\
\hline & & & & -721 & 8 & -721 & 7 & $\left.|0>\rightarrow| 52^{1} ; 1^{2}\right\rangle$ \\
\hline 865.7 & 11551 & -671 & $\sim 675$ & -685 & 94 & -685 & 91 & $\left.|0>\rightarrow| 52^{1}\right\rangle$ \\
\hline 862.9 & 11589 & -633 & & & & & & \\
\hline \multirow[t]{2}{*}{859.0} & 11641 & -581 & $\sim 580$ & -599 & 11 & -599 & 14 & $|0>\rightarrow| 45^{1}>$ \\
\hline & & & & & & -560 & 4 & $\left.|0>\rightarrow| 41^{1}\right\rangle$ \\
\hline 856.1 & 11681 & -541 & & -558 & 5 & -558 & 4 & $|0\rangle \rightarrow\left|29^{1} ; 17^{1}\right\rangle$ \\
\hline 851.2 & 11748 & -474 & $\sim 450$ & & & -487 & 14 & $\left.|0>\rightarrow| 35^{1}\right\rangle$ \\
\hline 848.8 & 11781 & -441 & & -451 & 6 & -451 & 5 & $\left.|0>\rightarrow| 17^{2}\right\rangle$ \\
\hline 841.8 & 11879 & -343 & & -353 & 8 & -353 & 7 & $\left.|0>\rightarrow| 17^{1} ; 13^{1}\right\rangle$ \\
\hline 835.7 & 11966 & -256 & & -259 & 13 & -259 & 10 & $\left.|0>\rightarrow| 24^{1}\right\rangle$ \\
\hline 832.9 & 12006 & -216 & & -243 & 14 & -243 & 12 & $|0\rangle \rightarrow\left|17^{1} ; 1^{1}\right\rangle$ \\
\hline \multirow[t]{2}{*}{828.2} & 12074 & -148 & & -158 & 26 & -158 & 18 & $\left.|0>\rightarrow| 14^{1}\right\rangle$ \\
\hline & & & & -146 & 18 & -146 & 15 & $\left.|0>\rightarrow| 13^{1} ; 1^{1}\right\rangle$ \\
\hline \multirow[t]{3}{*}{825.3} & 12117 & -105 & & -109 & 7 & -109 & 6 & $\left.|0>\rightarrow| 3^{2}\right\rangle$ \\
\hline & & & & -47 & 8 & -47 & 7 & $\left.|0>\rightarrow| 2^{1} ; 1^{1}\right\rangle$ \\
\hline & & & & -36 & 147 & -36 & 127 & $|0>\rightarrow| 1^{2}>$ \\
\hline 818.2 & 12222 & 0 & & 0 & 1863 & 0 & 1611 & $|0>\rightarrow| 0\rangle$ \\
\hline
\end{tabular}

${ }^{\mathrm{a}} \mathrm{v}=1 / \lambda \mathrm{Ne}$

${ }^{\text {b }} \mid 0>$ refers to vibrational ground state of $\mathrm{D}_{1}$; vibrational transitions of the ground state of $\mathrm{ZnPc}^{+}$calculated at B3LYP/cc-pVDZ level are listed in Table S1. 
Table S8. $\mathrm{S}_{1} \rightarrow \mathrm{S}_{0}$ fluorescence of $\mathrm{H}_{2} \mathrm{Pc}$. Comparison with (i) STM-LE measurements and (ii) predictions of TD-B3LYP/cc-pVDZ calculations at FC and FCHT levels (vibrotational assignments (final states mostly corresponding to single excitations)), excitation energies (in $\mathrm{cm}^{-1}$ ) and emission intensities).

\begin{tabular}{|c|c|c|c|c|c|}
\hline \multirow{3}{*}{$\begin{array}{c}\text { This work } \\
\mathrm{Ne} \\
\Delta \mathrm{v} / \mathrm{cm}^{-1 \mathrm{~b}}\end{array}$} & \multicolumn{4}{|c|}{ TD-B3LYP/cc-pVDZ } & \multirow[b]{3}{*}{ Assignment } \\
\hline & \multicolumn{2}{|r|}{ FC } & \multicolumn{2}{|c|}{ FCHT } & \\
\hline & $\Delta v / \mathrm{cm}^{-1}$ & Int. / $\mu \mathrm{J} \mathrm{mol}^{-1}$ & $\Delta v / \mathrm{cm}^{-1}$ & Int. / $\mu \mathrm{J} \mathrm{mol}^{-1}$ & \\
\hline & -1711 & 21 & -1711 & 21 & $|0>\rightarrow| 112^{1} ; 32^{1}>$ \\
\hline & -1691 & 15 & -1691 & 19 & $|0>\rightarrow| 142^{1} ; 3^{2}>$ \\
\hline \multirow[t]{3}{*}{-1617} & & & -1663 & 123 & $\left.|0>\rightarrow| 150^{1}\right\rangle$ \\
\hline & & & -1655 & 19 & $\left.|0>\rightarrow| 147^{1}\right\rangle$ \\
\hline & & & -1627 & 21 & $|0>\rightarrow| 144^{1}>$ \\
\hline \multirow[t]{5}{*}{-1546} & -1610 & 1101 & -1610 & 1403 & $|0>\rightarrow| 142^{1}>$ \\
\hline & & & -1587 & 284 & $|0>\rightarrow| 140^{1}>$ \\
\hline & & & -1512 & 62 & $\left.|0>\rightarrow| 136^{1}\right\rangle$ \\
\hline & -1502 & 20 & -1502 & 20 & $\left.|0>\rightarrow| 68^{1} ; 50^{1}\right\rangle$ \\
\hline & -1475 & 148 & -1477 & 767 & $\left.|0>\rightarrow| 131^{1}\right\rangle$ \\
\hline \multirow[t]{3}{*}{-1446} & -1430 & 19 & -1475 & 180 & $|0>\rightarrow| 130^{1}>$ \\
\hline & & & -1430 & 24 & $\left.|0>\rightarrow| 55^{1} ; 50^{1}\right\rangle$ \\
\hline & -1398 & 278 & -1398 & 347 & $|0>\rightarrow| 125^{1}>$ \\
\hline \multirow[t]{7}{*}{-1344} & -1385 & 193 & -1385 & 215 & $\left.|0>\rightarrow| 123^{1}\right\rangle$ \\
\hline & -1380 & 35 & -1380 & 35 & $|0>\rightarrow| 50^{2}>$ \\
\hline & & & -1329 & 676 & $\left.|0>\rightarrow| 118^{1}\right\rangle$ \\
\hline & & & -1309 & 344 & $\left.|0>\rightarrow| 116^{1}\right\rangle$ \\
\hline & & & -1306 & 22 & $\left.|0>\rightarrow| 112^{1} ; 3^{2}\right\rangle$ \\
\hline & & & -1256 & 100 & $\left.|0>\rightarrow| 113^{1}\right\rangle$ \\
\hline & & & -1225 & 1592 & $|0>\rightarrow| 112^{1}>$ \\
\hline \multirow[t]{3}{*}{-1184} & -1207 & 41 & -1207 & 64 & $\left.|0>\rightarrow| 108^{1}\right\rangle$ \\
\hline & & & -1177 & 18 & $|0>\rightarrow| 107^{1}>$ \\
\hline & & & -1176 & 21 & $\left.|0>\rightarrow| 50^{1} ; 32^{1}\right\rangle$ \\
\hline \multirow[t]{7}{*}{-1137} & -1154 & 187 & -1154 & 228 & $|0>\rightarrow| 104^{1}>$ \\
\hline & & & -1130 & 167 & $\left.|0>\rightarrow| 101^{1}\right\rangle$ \\
\hline & & & -1118 & 207 & $|0>\rightarrow| 99^{1}>$ \\
\hline & & & -1098 & 282 & $\left.|0>\rightarrow| 97^{1}\right\rangle$ \\
\hline & & & -1041 & 218 & $\left.|0>\rightarrow| 93^{1}\right\rangle$ \\
\hline & -1029 & 33 & -1029 & 38 & $\left.|0>\rightarrow| 92^{1}\right\rangle$ \\
\hline & & & -899 & 87 & $\left.|0>\rightarrow| 76^{1}\right\rangle$ \\
\hline \multirow[t]{3}{*}{-793} & -812 & 461 & -812 & 437 & $\left.|0>\rightarrow| 68^{1}\right\rangle$ \\
\hline & -782 & 33 & -782 & 31 & $\left.|0>\rightarrow| 61^{1}\right\rangle$ \\
\hline & -771 & 19 & & & $\left.|0>\rightarrow| 50^{1} ; 3^{2}\right\rangle$ \\
\hline-722 & -740 & 422 & -740 & 533 & $|0>\rightarrow| 55^{1}>$ \\
\hline \multirow[t]{2}{*}{-682} & -690 & 1385 & -690 & 1373 & $\left.|0>\rightarrow| 50^{1}\right\rangle$ \\
\hline & -578 & 25 & -578 & 24 & $\left.|0>\rightarrow| 25^{2}\right\rangle$ \\
\hline \multirow[t]{5}{*}{-568} & -576 & 169 & -576 & 158 & $|0>\rightarrow| 42^{1}>$ \\
\hline & & & -576 & 26 & $\left.|0>\rightarrow| 41^{1}\right\rangle$ \\
\hline & -550 & 57 & -550 & 60 & $\left.|0>\rightarrow| 38^{1}\right\rangle$ \\
\hline & -526 & 25 & -526 & 24 & $\left.|0>\rightarrow| 21^{2}\right\rangle$ \\
\hline & & & -486 & 456 & $\left.|0>\rightarrow| 32^{1}\right\rangle$ \\
\hline \multicolumn{6}{|l|}{-336} \\
\hline & -254 & 30 & -254 & 30 & $\left.|0>\rightarrow| 16^{1} ; 3^{1}\right\rangle$ \\
\hline \multirow[t]{2}{*}{-217} & -230 & 35 & -230 & 34 & $\left.|0>\rightarrow| 19^{1}\right\rangle$ \\
\hline & & & -178 & 153 & $\left.|0>\rightarrow| 14^{1}\right\rangle$ \\
\hline \multirow[t]{2}{*}{-119} & -116 & 39 & -116 & 38 & $\left.|0>\rightarrow| 5^{2}\right\rangle$ \\
\hline & -81 & 409 & -81 & 396 & $\left.|0>\rightarrow| 3^{2}\right\rangle$ \\
\hline \multirow[t]{3}{*}{-64} & & & -80 & 57 & $\left.|0>\rightarrow| 6^{1}\right\rangle$ \\
\hline & -38 & 93 & -38 & 90 & $\left.|0>\rightarrow| 1^{2}\right\rangle$ \\
\hline & 0 & 30230 & 0 & 29290 & $|0>\rightarrow| 0\rangle$ \\
\hline
\end{tabular}

${ }^{\mathrm{a}} \mathrm{v}=1 / \lambda \mathrm{Ne}$

${ }^{\mathrm{b}} \mid 0>$ refers to vibrational ground state of $\mathrm{D}_{1}$; vibrational transitions of the ground state of $\mathrm{ZnPc}^{+}$calculated at B3LYP/cc-pVDZ level are listed in Table S1. 


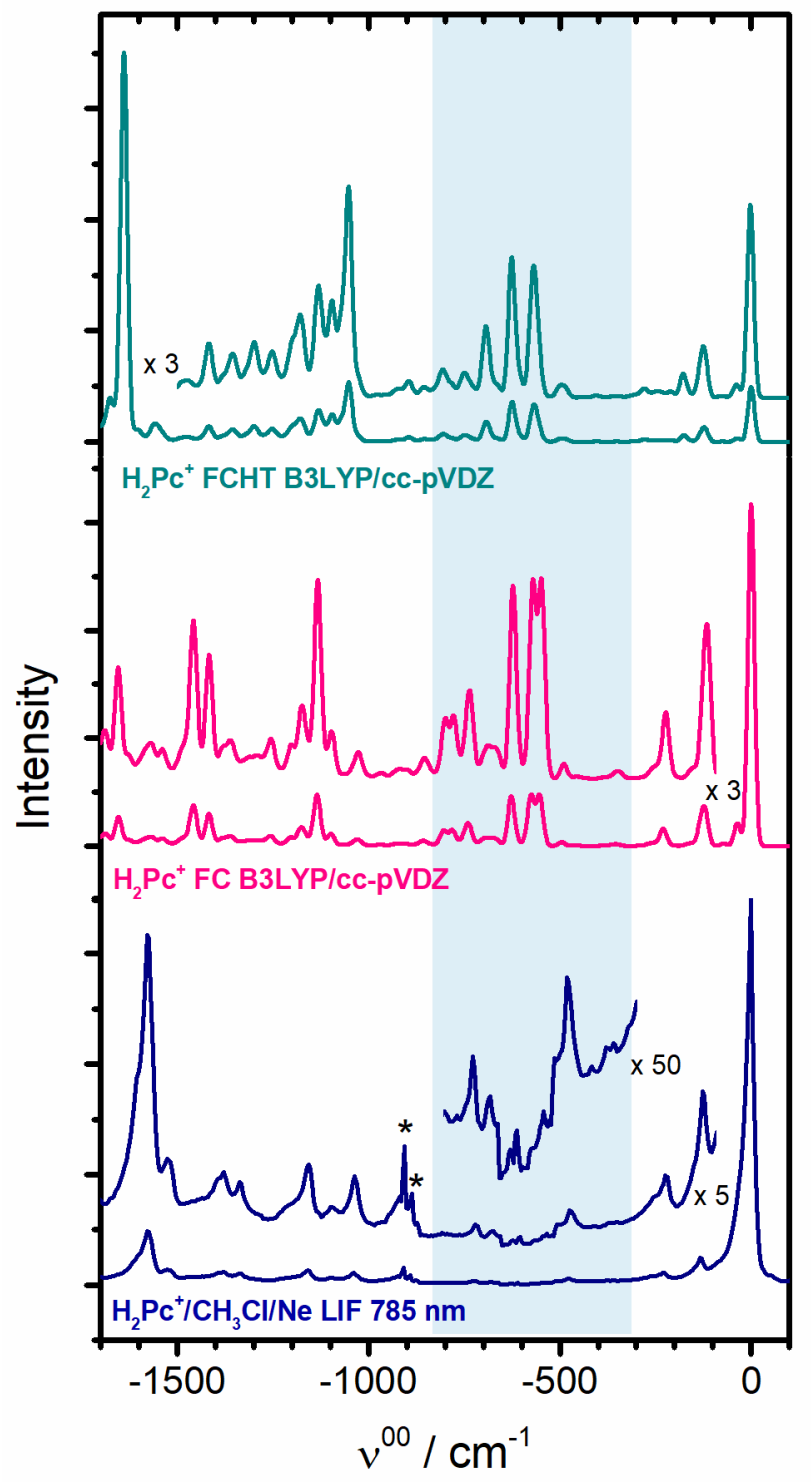

Figure S9. Comparison of the LIF spectrum of $\mathrm{H}_{2} \mathrm{Pc}^{+}$recorded in a $\mathrm{H}_{2} \mathrm{Pc}^{+} / \mathrm{CH}_{3} \mathrm{Cl} / \mathrm{Ne}$ matrix ( $785 \mathrm{~nm}$ excitation) with TD-B3LYP/cc-pVDZ (FCHT) and TD-B3PW91/cc-pVDZ (FC) predictions. Blue area indicates the region from $910-920 \mathrm{~nm}$ for which intensity calibration was subject to larger than standard errors (Note, that this issue was not relevant for the other three species probed here - whose emission is further to the blue). * Indicates lines of an unknown impurity which do not correlate in intensities with lines of $\mathrm{H}_{2} \mathrm{Pc}^{+}$. 
Table S9. $\mathrm{D}_{1} \rightarrow \mathrm{D}_{0}$ fluorescence of $\mathrm{H}_{2} \mathrm{Pc}^{+}$. Tabulation of vibrotational features observed in the LIF spectrum of matrix isolated $\mathrm{H}_{2} \mathrm{Pc}^{+}$. We do not attempt an assignment due to unsatisfactory description by theory (see Table S10).

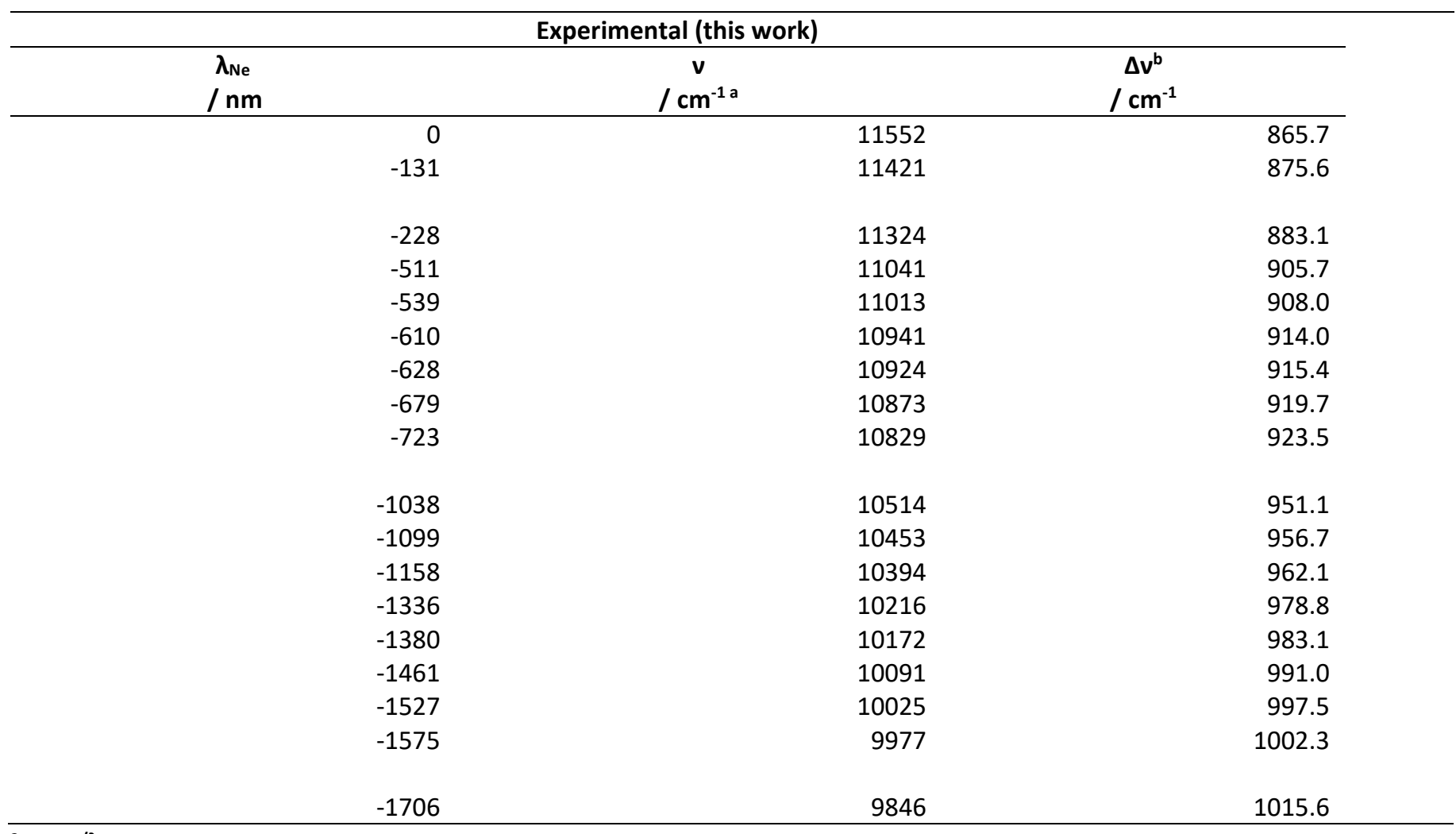

${ }^{\mathrm{a}} v=1 / \lambda_{\mathrm{Ne}}$

${ }^{b}$ Wavenumber difference in respect to $0-0$ transition.

${ }^{c}|0\rangle$ refers to the vibrational ground state of $\mathrm{D}_{1}$; The vibrational wavenumbers of the ground state of $\mathrm{H}_{2} \mathrm{Pc}^{+}$calculated at B3LYP/cc-pVDZ are listed in Table S1. 
Table S10. $\mathrm{D}_{1} \rightarrow \mathrm{D}_{0}$ fluorescence of $\mathrm{H}_{2} \mathrm{Pc}^{+}$. Tabulation of vibrational features predicted by FC and FCHT TDB3LYP/cc-pVDZ method simulations (in $\mathrm{cm}^{-1}$ ), emission intensities, and assignments.

\begin{tabular}{|c|c|c|c|c|c|}
\hline \multirow{3}{*}{$\begin{array}{c}\text { This work } \\
\text { Ne } \\
\Delta v / \mathrm{cm}^{-1 \mathrm{~b}}\end{array}$} & \multicolumn{4}{|c|}{ TD-B3LYP/cc-pVDZ } & \multirow[b]{3}{*}{ Assignment } \\
\hline & \multicolumn{2}{|c|}{ FC } & \multicolumn{2}{|c|}{ FCHT } & \\
\hline & $\Delta v / \mathrm{cm}^{-1}$ & Int. / $\mu \mathrm{J} \mathrm{mol}^{-1}$ & $\Delta v / \mathrm{cm}^{-1}$ & Int. / $\mu \mathrm{J} \mathrm{mol}^{-1}$ & \\
\hline & -1711 & 21 & -1711 & 21 & $|0>\rightarrow| 112^{1} ; 32^{1}>$ \\
\hline & -1691 & 15 & -1691 & 19 & $|0>\rightarrow| 142^{1} ; 3^{2}>$ \\
\hline \multirow[t]{3}{*}{-1617} & & & -1663 & 123 & $|0>\rightarrow| 150^{1}>$ \\
\hline & & & -1655 & 19 & $|0>\rightarrow| 147^{1}>$ \\
\hline & & & -1627 & 21 & $|0>\rightarrow| 144^{1}>$ \\
\hline \multirow[t]{5}{*}{-1546} & -1610 & 1101 & -1610 & 1403 & $\left.|0>\rightarrow| 142^{1}\right\rangle$ \\
\hline & & & -1587 & 284 & $\left.|0>\rightarrow| 140^{1}\right\rangle$ \\
\hline & & & -1512 & 62 & $|0>\rightarrow| 136^{1}>$ \\
\hline & -1502 & 20 & -1502 & 20 & $\left.|0>\rightarrow| 68^{1} ; 50^{1}\right\rangle$ \\
\hline & -1475 & 148 & -1477 & 767 & $\left.|0>\rightarrow| 131^{1}\right\rangle$ \\
\hline \multirow[t]{3}{*}{-1446} & -1430 & 19 & -1475 & 180 & $|0>\rightarrow| 130^{1}>$ \\
\hline & & & -1430 & 24 & $\left.|0>\rightarrow| 55^{1} ; 50^{1}\right\rangle$ \\
\hline & -1398 & 278 & -1398 & 347 & $|0>\rightarrow| 125^{1}>$ \\
\hline \multirow[t]{7}{*}{-1344} & -1385 & 193 & -1385 & 215 & $\left.|0>\rightarrow| 123^{1}\right\rangle$ \\
\hline & -1380 & 35 & -1380 & 35 & $\left.|0>\rightarrow| 50^{2}\right\rangle$ \\
\hline & & & -1329 & 676 & $\left.|0>\rightarrow| 118^{1}\right\rangle$ \\
\hline & & & -1309 & 344 & $\left.|0>\rightarrow| 116^{1}\right\rangle$ \\
\hline & & & -1306 & 22 & $\left.|0>\rightarrow| 112^{1} ; 3^{2}\right\rangle$ \\
\hline & & & -1256 & 100 & $|0>\rightarrow| 113^{1}>$ \\
\hline & & & -1225 & 1592 & $\left.|0>\rightarrow| 112^{1}\right\rangle$ \\
\hline \multirow[t]{3}{*}{-1184} & -1207 & 41 & -1207 & 64 & $\left.|0>\rightarrow| 108^{1}\right\rangle$ \\
\hline & & & -1177 & 18 & $|0>\rightarrow| 107^{1}>$ \\
\hline & & & -1176 & 21 & $\left.|0>\rightarrow| 50^{1} ; 32^{1}\right\rangle$ \\
\hline \multirow[t]{7}{*}{-1137} & -1154 & 187 & -1154 & 228 & $|0>\rightarrow| 104^{1}>$ \\
\hline & & & -1130 & 167 & $\left.|0>\rightarrow| 101^{1}\right\rangle$ \\
\hline & & & -1118 & 207 & $\left.|0>\rightarrow| 99^{1}\right\rangle$ \\
\hline & & & -1098 & 282 & $|0>\rightarrow| 97^{1}>$ \\
\hline & & & -1041 & 218 & $\left.|0>\rightarrow| 93^{1}\right\rangle$ \\
\hline & -1029 & 33 & -1029 & 38 & $\left.|0>\rightarrow| 92^{1}\right\rangle$ \\
\hline & & & -899 & 87 & $\left.|0>\rightarrow| 76^{1}\right\rangle$ \\
\hline \multirow[t]{3}{*}{-793} & -812 & 461 & -812 & 437 & $\left.|0>\rightarrow| 68^{1}\right\rangle$ \\
\hline & -782 & 33 & -782 & 31 & $\left.|0>\rightarrow| 61^{1}\right\rangle$ \\
\hline & -771 & 19 & & & $\left.|0>\rightarrow| 50^{1} ; 3^{2}\right\rangle$ \\
\hline-722 & -740 & 422 & -740 & 533 & $|0>\rightarrow| 55^{1}>$ \\
\hline \multirow[t]{2}{*}{-682} & -690 & 1385 & -690 & 1373 & $\left.|0>\rightarrow| 50^{1}\right\rangle$ \\
\hline & -578 & 25 & -578 & 24 & $|0>\rightarrow| 25^{2}>$ \\
\hline \multirow[t]{5}{*}{-568} & -576 & 169 & -576 & 158 & $|0>\rightarrow| 42^{1}>$ \\
\hline & & & -576 & 26 & $\left.|0>\rightarrow| 41^{1}\right\rangle$ \\
\hline & -550 & 57 & -550 & 60 & $\left.|0>\rightarrow| 38^{1}\right\rangle$ \\
\hline & -526 & 25 & -526 & 24 & $\left.|0>\rightarrow| 21^{2}\right\rangle$ \\
\hline & & & -486 & 456 & $\left.|0>\rightarrow| 32^{1}\right\rangle$ \\
\hline \multicolumn{6}{|l|}{-336} \\
\hline & -254 & 30 & -254 & 30 & $\left.|0>\rightarrow| 16^{1} ; 3^{1}\right\rangle$ \\
\hline \multirow[t]{2}{*}{-217} & -230 & 35 & -230 & 34 & $|0>\rightarrow| 19^{1}>$ \\
\hline & & & -178 & 153 & $\left.|0>\rightarrow| 14^{1}\right\rangle$ \\
\hline \multirow[t]{2}{*}{-119} & -116 & 39 & -116 & 38 & $\left.|0>\rightarrow| 5^{2}\right\rangle$ \\
\hline & -81 & 409 & -81 & 396 & $\left.|0>\rightarrow| 3^{2}\right\rangle$ \\
\hline \multirow[t]{3}{*}{-64} & & & -80 & 57 & $\left.|0>\rightarrow| 6^{1}\right\rangle$ \\
\hline & -38 & 93 & -38 & 90 & $\left.|0>\rightarrow| 1^{2}\right\rangle$ \\
\hline & 0 & 30230 & 0 & 29290 & $|0>\rightarrow| 0>$ \\
\hline
\end{tabular}

${ }^{\mathrm{a}} \mathrm{v}=1 / \lambda \mathrm{Ne}$

${ }^{b} \mid 0>$ refers to vibrational ground state of $D_{1}$; vibrational transitions of the ground state of ZnPc ${ }^{+}$calculated at B3LYP/cc-pVDZ level are listed in Table S1. 


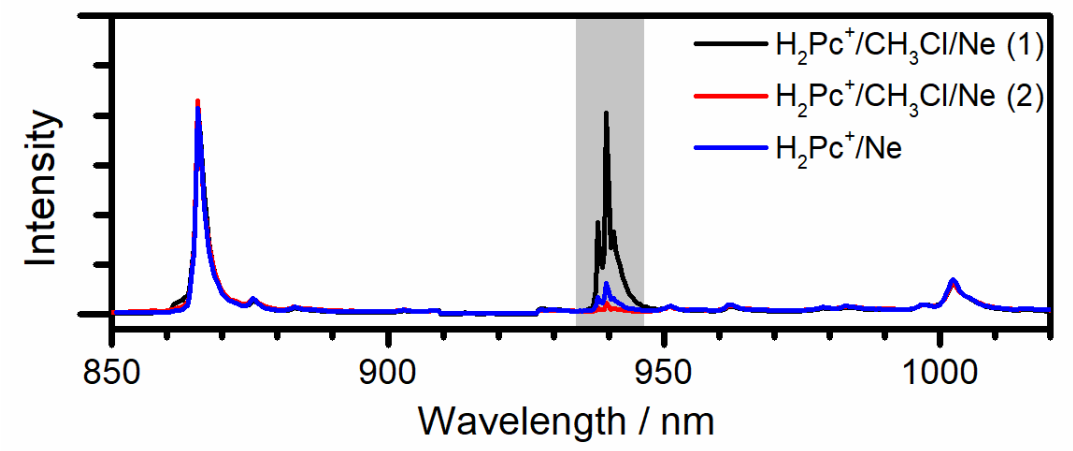

Figure S10. Comparison of the LIF spectrum of $\mathrm{H}_{2} \mathrm{Pc}^{+}$recorded in an $\mathrm{H}_{2} \mathrm{Pc}^{+} / \mathrm{CH}_{3} \mathrm{Cl} / \mathrm{Ne}$ at 2 different focal points and $\mathrm{H}_{2} \mathrm{Pc}^{+} / \mathrm{Ne}$ matrices (785 $\mathrm{nm}$ excitation). The Gray area indicates the region of unknown impurity.

Table S11. Comparison of the band origins of $\mathrm{ZnPc}, \mathrm{ZnPc}^{+}, \mathrm{H}_{2} \mathrm{Pc}$, and $\mathrm{H}_{2} \mathrm{Pc}^{+}$as observed here in absorption and emission spectra with predictions from TD-B3LYP/cc-pVDZ method calculations.

\begin{tabular}{|c|c|c|c|c|c|c|c|c|c|}
\hline \multirow[b]{3}{*}{ Abs. } & & \multicolumn{2}{|c|}{$\mathrm{H}_{2} \mathrm{Pc}$} & \multicolumn{2}{|c|}{$\mathrm{ZnPc}$} & \multicolumn{2}{|c|}{$\mathrm{H}_{2} \mathrm{Pc}^{+}$} & \multicolumn{2}{|c|}{$\mathrm{ZnPc}^{+}$} \\
\hline & & Exp. & Calc. & Exp. & Calc. & Exp. & Calc. & Exp. & Calc. \\
\hline & $Q_{x}$ & $14984 \pm 5$ & 15653 & $15589 \pm 5$ & 15808 & $11546 \pm 3$ & 11313 & $12153 \pm 3$ & 12153 \\
\hline & $Q_{r}$ & $16025 \pm 5$ & 15735 & & & $11947 \pm 3$ & & & \\
\hline LIF & & $15015 \pm 5$ & 15735 & $15557 \pm 5$ & 15808 & $11549 \pm 3$ & 11313 & $12222 \pm 3$ & 12153 \\
\hline
\end{tabular}

